JOURNAL OF THE

AMERICAN MATHEMATICAL SOCIETY

Volume 11, Number 4, October 1998, Pages 869-897

S 0894-0347(98)00280-X

\title{
RADON TRANSFORMS AND FINITE TYPE CONDITIONS
}

\author{
ANDREAS SEEGER
}

\section{INTRODUCTION}

The purpose of this paper is to study averaging operators of Radon transform type. We shall formulate suitable finite type conditions and prove $L^{p}$-Sobolev and $L^{p} \rightarrow L^{q}$ estimates. The results will be essentially sharp for operators associated with families of curves in the plane.

Let $\mathfrak{X}$ and $\mathfrak{Y}$ be smooth manifolds, $\operatorname{dim} \mathfrak{X}=n_{L}, \operatorname{dim} \mathfrak{Y}=n_{R}$, and let $\mathcal{M}$ be a submanifold in $\mathfrak{X} \times \mathfrak{Y}$ with conormal bundle $N^{*} \mathcal{M}$; we denote by $\ell$ the codimension of $\mathcal{M}$. We shall always assume that the projections $\pi_{\mathfrak{X}}: \mathcal{M} \rightarrow \mathfrak{X}$ and $\pi_{\mathfrak{Y}}: \mathcal{M} \rightarrow \mathfrak{Y}$ are surjective with $\operatorname{rank} D \pi_{\mathfrak{X}}=n_{L}$, rank $D \pi_{\mathfrak{Y}}=n_{R}$. This in particular implies that $N^{*} \mathcal{M} \subset T^{*} \mathfrak{X} \backslash 0 \times T^{*} \mathfrak{Y} \backslash 0$ where 0 refers to the zero sections in $T^{*} \mathfrak{X}$ and $T^{*} \mathfrak{Y}$, respectively. This is the usual assumption for the canonical relation associated with Lagrangian distributions arising as kernels of Fourier integral operators.

The assumptions on $D \pi_{\mathfrak{X}}, D \pi_{\mathfrak{Y}}$ imply that for fixed $x \in \mathfrak{X}, y \in \mathfrak{Y}$ the sets

$$
\begin{aligned}
& \mathcal{M}_{x}=\{y \in \mathfrak{Y} ;(x, y) \in \mathcal{M}\}, \\
& \mathcal{M}^{y}=\{x \in \mathfrak{X} ;(x, y) \in \mathcal{M}\}
\end{aligned}
$$

are smooth immersed submanifolds of codimension $\ell$ in $\mathfrak{Y}$ and $\mathfrak{X}$, respectively.

Let $\chi \in C^{\infty}(\mathfrak{X} \times \mathfrak{Y})$ be compactly supported. We shall study the regularity of the averaging operator (or generalized Radon transform) given by

$$
R f(x)=\int_{\mathcal{M}_{x}} \chi(x, y) f(y) d \sigma_{x}(y) ;
$$

here $d \sigma_{x}$ is a smooth density on $\mathcal{M}_{x}$ depending smoothly on $x$.

The averaging operator $R$ is a Fourier integral operator and its distribution kernel belongs to the class $I^{m}\left(\mathfrak{X}, \mathfrak{Y}, N^{*} \mathcal{M}\right)$ with $m=\ell / 2-\left(n_{L}+n_{R}\right) / 4$ (cf. [10]). Sharp estimates are well known in the case where the projections $\pi_{L}: N^{*} \mathcal{M} \rightarrow T^{*} \mathfrak{X}$, $\pi_{R}: N^{*} \mathcal{M} \rightarrow T^{*} \mathfrak{Y}$ are locally diffeomorphisms $([10])$; then necessarily $n_{L}=n_{R}$. There has been considerable interest in obtaining sharp estimates in the degenerate case where this assumption is relaxed (see e.g. [4], [5], [6], [7], [8], [12], [13], [14], [15], [16], [17], [19], [20], [21]).

Finite type conditions may be formulated in terms of vector fields tangent to $\mathcal{M}$ and their commutators. Such conditions came up in the work by Hörmander [9], Kohn [11], Rothschild and Stein [18]) on subelliptic estimates. Later, around 1985, Christ, Nagel, Stein and Wainger (see [3]) used them to investigate $L^{p}$ boundedness

Received by the editors October 28, 1997.

1991 Mathematics Subject Classification. Primary 35S30; Secondary 47G10, 32F40, 44A12.

Key words and phrases. Averaging operators, Radon transforms, finite type conditions.

The author's research was supported in part by an NSF grant.

(C)1998 American Mathematical Society 
of singular Radon transforms. This work dealt with the situation $\mathfrak{X}=\mathfrak{Y}$, and $\mathcal{M}$ contains the diagonal in $\mathfrak{X} \times \mathfrak{X}$; then the manifold $\mathcal{M}_{x}$ in (1.1) can be parametrized by $t \mapsto \gamma(x, t)$, with $\gamma(x, 0)=x$. It was shown that there exist unique vector fields $U_{\alpha}$ so that $\gamma(x, t) \sim \exp \left(\sum_{\alpha} \frac{t^{\alpha} U_{\alpha}}{\alpha !}\right)$ as $t \rightarrow 0$; the "curvature" or finite type condition is then that the $U_{\alpha}$ together with their iterated commutators span the tangent space at every $P \in \mathfrak{X}$. It is also shown in [3] that this condition is also equivalent with an $L^{2} \rightarrow L_{\varepsilon}^{2}$ smoothing estimate for averaging operators, for some $\varepsilon>0$. However it seems quite hard to obtain sharp regularity theorems using an expansion in terms of the $U_{\alpha}$; the relation between the singularities of the projections $\pi_{L}$ and $\pi_{R}$ and the $U_{\alpha}$ is not well understood. For the (simplest) case of fold singularities on the diagonal see Phong and Stein [15].

In the present paper we formulate finite type conditions which lead modulo endpoints to a complete description of the regularity properties at least in 2 dimensions. Here we emphasize the distinction between vector fields tangent to both $\mathcal{M}$ and $\mathfrak{X}$ and vector fields tangent to $\mathcal{M}$ and $\mathfrak{Y}$; this distinction is crucial for the derivation of precise regularity results. We therefore introduce the subspaces

$$
\begin{aligned}
& T_{P}^{1,0} \mathcal{M}=T_{P} \mathcal{M} \cap T_{P}(\mathfrak{X} \times\{0\}), \\
& T_{P}^{0,1} \mathcal{M}=T_{P} \mathcal{M} \cap T_{P}(\{0\} \times \mathfrak{Y}) ;
\end{aligned}
$$

so if $P=(x, y) \in \mathcal{M}$, then $T_{P}^{1,0} \mathcal{M}$ is tangent to $\mathcal{M}^{y} \times\{0\}$ and $T_{P}^{0,1} \mathcal{M}$ is tangent to $\{0\} \times \mathcal{M}_{x}$. Note that $\operatorname{dim} T_{P}^{1,0} \mathcal{M}=n_{L}-\ell, \operatorname{dim} T_{P}^{0,1} \mathcal{M}=n_{R}-\ell$ and $\operatorname{dim} T_{P} \mathcal{M}=$ $n_{L}+n_{R}-\ell$. The finite type conditions will measure to which extent the subbundle $T^{1,0} \mathcal{M} \oplus T^{0,1} \mathcal{M}$ fails to be involutive.

Given the calculus of wavefront sets ([10]) it is also natural to formulate finite type conditions in terms of the twisted normal bundle or canonical relation

$$
\mathcal{C}=\left(N^{*} \mathcal{M}\right)^{\prime}=\left\{(x, \xi, y,-\eta):(x, \xi, y, \eta) \in N^{*} \mathcal{M}\right\} .
$$

In fact $\left(N^{*} \mathcal{M}\right)^{\prime}$ can be identified with a subbundle $T^{*, \perp} \mathcal{M}$ of $T^{*} \mathcal{M}$ whose fiber at $P=(x, y) \in \mathcal{M}$ is

$$
T_{P}^{*, \perp} \mathcal{M}=\left(T_{P}^{1,0} \mathcal{M} \oplus T_{P}^{0,1} \mathcal{M}\right)^{\perp},
$$

the $\ell$-dimensional space of all linear functionals in $T_{P}^{*} \mathcal{M}$ which annihilate vectors in $T_{P}^{1,0} \mathcal{M}$ and vectors in $T_{P}^{0,1} \mathcal{M}$. The identification is via restricting linear forms in $T_{P}^{*}(\mathfrak{X} \times \mathfrak{Y})$ to tangent vectors in $T_{P} \mathcal{M}$. More precisely if $\imath: \mathcal{M} \rightarrow \mathfrak{X} \times \mathfrak{Y}$ denotes the inclusion map and $\imath^{*}$ the pullback of $i$ acting on forms in $T^{*}(\mathfrak{X} \times \mathfrak{Y})$, then

$$
T^{*, \perp} \mathcal{M}=\left\{\left(P, \imath_{P}^{*} \lambda\right):(P, \lambda) \in \mathcal{C}\right\}
$$

where $P=(x, y) \in \mathcal{M}$ and $\lambda=(\xi,-\eta) \in\left(N_{P}^{*} \mathcal{M}\right)^{\prime}$; see the discussion in $\S 2$.

Given vector fields $V, W$ we denote the Lie-derivative of $W$ with respect to $V$ (or Lie product of $V$ and $W$ ) by [V,W]; in coordinates it is identified with the commutator of the vector fields $V$ and $W$. As customary in Lie-theory we shall also use the notation $\operatorname{ad} V(W):=[V, W]$. We first introduce the notion of a vector field of type $\preceq(\mu, \nu)$. Here $\prec$ refers to the partial order on pairs of integers defined by $(j, k) \prec\left(j^{\prime}, k^{\prime}\right)$ if and only if either $j \leq j^{\prime}$ and $k<k^{\prime}$ or $j<j^{\prime}$ and $k \leq k^{\prime}$. We say that $(j, k) \preceq\left(j^{\prime}, k^{\prime}\right)$ if $(j, k) \prec\left(j^{\prime}, k^{\prime}\right)$ or $(j, k)=\left(j^{\prime}, k^{\prime}\right)$.

Definition. Let $\mathcal{U}$ be an open set in $\mathcal{M}$.

(i) A vector field $V$ is of type $(1,0)$ in $\mathcal{U}$ if $V_{P} \in T_{P}^{1,0} \mathcal{M}$ for all $P \in \mathcal{U}$.

(ii) A vector field $V$ is of type $(0,1)$ in $\mathcal{U}$ if $V_{P} \in T_{P}^{0,1} \mathcal{M}$ for all $P \in \mathcal{U}$. 
(iii) We set $\mathcal{V}^{(0,0)}(\mathcal{U})=\{0\}$ and let $\mathcal{V}^{1,0}, \mathcal{V}^{0,1}$ be the $C^{\infty}(\mathcal{U})$-modules of all vector fields of type $(1,0),(0,1)$, respectively. For a pair $(\mu, \nu)$ of positive integers let $\mathcal{V}^{\mu, \nu}(\mathcal{U})$ be the $C^{\infty}(\mathcal{U})$-module generated by all vector fields of type $(1,0)$ and $(0,1)$ and all vector fields of the form $g \operatorname{ad} V_{1} \cdots \operatorname{ad} V_{n-1}\left(V_{n}\right)$, where $g \in C^{\infty}(\mathcal{U})$, each $V_{i}$ is of type $(1,0)$ or of type $(0,1)$, at most $\mu$ of the $V_{i}$ are of type $(1,0)$ and at most $\nu$ of the $V_{i}$ are of type $(0,1)$.

(iv) If $V \in \mathcal{V}^{\mu, \nu}(\mathcal{U})$, then we say that $V$ is of type $\preceq(\mu, \nu)$ in $\mathcal{U}$. If $V \in \mathcal{V}^{\mu, \nu}(\mathcal{U})$ but $V \notin \mathcal{V}^{\mu^{\prime}, \nu^{\prime}}(\mathcal{U})$ for any $\left(\mu^{\prime}, \nu^{\prime}\right) \prec(\mu, \nu)$, then we say that $V$ is of type $(\mu, \nu)$ in $\mathcal{U}$.

(v) Let $P \in \mathcal{M}$. Two vector fields $V$ and $\widetilde{V}$ are said to be equivalent at $P$ if there is a neighborhood $\mathcal{U}$ so that $V$ and $\widetilde{V}$ coincide in $\mathcal{U}$. The set of equivalence classes of vector fields (or germs) at $P$ is denoted by $\mathcal{V}(P)$. The submodule $\mathcal{V}^{\mu, \nu}(P)$ consists of the equivalence classes for which any representative is in $\mathcal{V}^{\mu, \nu}(\mathcal{U})$, for some suitable $\mathcal{U}$. By abuse of notation we shall say that a vector field $V$ defined on $\mathcal{M}$ near $P$ belongs to $\mathcal{V}^{\mu, \nu}(P)$ if $V \in \mathcal{V}^{\mu, \nu}(\mathcal{U})$ for some neighborhood $\mathcal{U}$ of $P$.

We remark that the notion of type $(\mu, \nu)$ is invariant under special changes of variables of the form $\Psi(x, y)=\left(\Psi_{L}(x), \Psi_{R}(y)\right)$ where $\Psi_{L}$ is a diffeomorphism in $\mathfrak{X}$ and $\Psi_{R}$ is a diffeomorphism in $\mathfrak{Y}$. Of course those are just the changes of variables which leave the $L^{p}$-Sobolev mapping properties of an operator invariant.

To see the invariance let $\Psi^{*} V$ be the pullback of $V$ under $\Psi$ defined by $\left(\Psi^{*} V\right)_{P}=$ $(D \Psi)_{P}^{-1} V_{\Psi(P)}$. Then the pullback of a vector field of type $(1,0)$ is of type $(1,0)$ (with respect to the manifold $\Psi^{-1}(\mathcal{M})$ ), in fact $\left(\Psi^{*} X\right)_{P}=\left(D \Psi_{L} \times \mathrm{Id}\right)_{P}^{-1} X_{\Psi(P)}$ for $P \in \Psi^{-1} \mathcal{M}$. Similarly, the pullback of a vector field of type $(0,1)$ is of type $(0,1)$. Now $\Psi^{*}[X, Y]=\left[\Psi^{*} X, \Psi^{*} Y\right]$ and the latter equals $\left[\Psi_{L}^{*} X, \Psi_{R}^{*} Y\right]$, which is a vector field of type $(1,1)$. Inductively we see that the notion of a vector field of type $(\mu, \nu)$ is invariant under change of variables in $\mathfrak{X}$ and $\mathfrak{Y}$.

Definition. Let $P \in \mathcal{M}$ and $\lambda \in T_{P}^{*} \mathcal{M}$. Then $T^{*} \mathcal{M}$ is said to be of type $(\mu, \nu)$ at $(P, \lambda)$ if there is $V \in \mathcal{V}^{\mu, \nu}(P)$ such that $\left\langle\lambda, V_{P}\right\rangle \neq 0$ and if $\left\langle\lambda, \widetilde{V}_{P}\right\rangle=0$ for all vector fields $\widetilde{V}$ of type $\prec(\mu, \nu)$. $\mathcal{M}$ is said to be of finite type at $P$ if for every $\lambda \in T_{P}^{*} \mathcal{M}$ there are nonnegative integers $\mu, \nu$ so that $T^{*} \mathcal{M}$ is of type $(\mu, \nu)$ at $(P, \lambda)$.

By the above comments on the behavior of vector fields of type $(\mu, \nu)$ under separate changes of variables in $\mathfrak{X}$ and $\mathfrak{Y}$ it is clear that the notion of type $(\mu, \nu)$ is well defined. Note that $T^{*} \mathcal{M}$ is always of type $(1,0)$ at $(P, \lambda)$ if $\lambda$ does not annihilate vectors in $T_{P}^{1,0} \mathcal{M}$, and of type $(0,1)$ if $\lambda$ does not annihilate vectors in $T_{P}^{0,1} \mathcal{M}$. Thus the finite type condition is only interesting if restricted to $T^{*, \perp} \mathcal{M}$. We note that the type is semicontinuous with respect to the partial order $\prec$; in the sense that if $T^{*} \mathcal{M}$ is of type $(\mu, \nu)$ at $(P, \lambda)$, then there is a neighborhood of $(P, \lambda)$ in $T^{*} \mathcal{M}$, conic in the fiber, such that $T^{*} \mathcal{M}$ is of type $\preceq(\mu, \nu)$ in this neighborhood. The number

$$
n(P)=\max _{\lambda \in T_{P}^{*} \mathcal{M}} \min \left\{\mu+\nu: T^{*} \mathcal{M} \text { is of finite type }(\mu, \nu) \text { at }(P, \lambda)\right\}
$$

is called the Hörmander type. This terminology is suggested by conditions in [9]: If $n=n(P)<\infty$, then there are numbers $\mu_{i}, \nu_{i}$, with $\mu_{i}+\nu_{i} \leq n$ and vector fields 
$V_{i}$ of type $\left(\mu_{i}, \nu_{i}\right), i=1, \ldots, n_{L}+n_{R}-\ell$, so that the $\left(V_{i}\right)_{P}$ span the tangent space $T_{P} \mathcal{M}{ }^{1}$

In this paper we emphasize the case $\operatorname{codim} \mathcal{M}=1$, so that $\mathcal{M}$ is a hypersurface in $\mathfrak{X} \times \mathfrak{Y}$ and the fibers of $T^{*, \perp} \mathcal{M}$ are one-dimensional. Then $T^{*} \mathcal{M}$ is of type $(\mu, \nu)$ at $(P, \lambda)$ for one $\lambda \in\left(T_{P}^{1,0} \mathcal{M} \oplus T_{P}^{0,1} \mathcal{M}\right)^{\perp}$ if and only if it is of type $(\mu, \nu)$ for all nonzero $\lambda \in\left(T_{P}^{1,0} \mathcal{M} \oplus T_{P}^{0,1} \mathcal{M}\right)^{\perp}$. This justifies the following terminology.

Definition. Suppose that $\mathcal{M}$ is a hypersurface in $\mathfrak{X} \times \mathfrak{Y}$, so that the projections to $\mathfrak{X}$ and $\mathfrak{Y}$ are submersions. Let $P \in \mathcal{M}, \mu \geq 1, \nu \geq 1$. Then $\mathcal{M}$ is said to be of type $(\mu, \nu)$ at $P$ if there is a vector field $V$ of type $(\mu, \nu)$ defined near $P$ and a linear functional $\lambda \in T_{P}^{*} \mathcal{M}$ annihilating vectors in $T_{P}^{1,0} \mathcal{M}$ and vectors in $T_{P}^{0,1} \mathcal{M}$ so that

$$
\left\langle\lambda, V_{P}\right\rangle \neq 0 .
$$

Again $\mathcal{M}$ is said to be of finite type at $P$ if $\mathcal{M}$ is of type $(\mu, \nu)$ at $P$, for a pair $(\mu, \nu)$ of positive integers.

We shall now formulate sharp theorems for curves in two-dimensional manifolds and generalizations for hypersurfaces $\mathcal{M}$. For every $P \in \mathcal{M}$ let

$$
\tau(P)=\left\{(\mu, \nu) \in \mathbb{Z}_{+} \times \mathbb{Z}_{+}: \mathcal{M} \text { is of type }(\mu, \nu) \text { at } P\right\} .
$$

Let $\overline{\mathcal{A}}(P)$ be the closed convex hull of the points in $\{(u, \beta): 0 \leq u \leq 1, \beta \leq 0\}$ and the points

$$
\left(\frac{\mu}{\mu+\nu}, \frac{1}{\mu+\nu}\right), \quad(\mu, \nu) \in \tau(P) .
$$

Let $\mathcal{A}(P)$ be the closed convex hull of the points in $\{(u, \beta): 0 \leq u \leq 1, \beta \leq 0\}$ and the points

Notice that

$$
\left(\frac{\frac{\mu}{n_{L}-1}}{\frac{\mu}{n_{L}-1}+\frac{\nu}{n_{R}-1}}, \frac{1}{\frac{\mu}{n_{L}-1}+\frac{\nu}{n_{R}-1}}\right), \quad(\mu, \nu) \in \tau(P) .
$$

$$
\overline{\mathcal{A}}(P)=\underline{\mathcal{A}}(P) \quad \text { if } n_{L}=n_{R}=2 .
$$

Theorem 1.1. Suppose that $\operatorname{codim} \mathcal{M}=1, P \in \mathcal{M}$ and $1 \leq p \leq \infty$.

(i) There is a neighborhood $\mathcal{U}$ of $P$ such that if supp $\chi \subset \mathcal{U}$ and if $(1 / p, \alpha)$ belongs to the interior of $\overline{\mathcal{A}}(P)$, then $R$ is bounded from $L_{s, \mathrm{comp}}^{p}(\mathfrak{Y})$ into $L_{s+\alpha, \text { loc }}^{p}(\mathfrak{X})$, for all $s \in \mathbb{R}$.

(ii) Suppose that $R$ is bounded from $L_{s, \mathrm{comp}}^{p}(\mathfrak{Y})$ into $L_{s+\alpha, \text { loc }}^{p}(\mathfrak{X})$, for some $s \in \mathbb{R}$, and that $\chi(P) \neq 0$. Then $(1 / p, \alpha)$ belongs to $\underline{\mathcal{A}}(P)$.

We note that since supp $\chi$ is compact we are working with functions of compact support, and the distinction between the spaces $L_{\alpha}^{p}, L_{\alpha, \text { comp }}^{p}, L_{\alpha, \text { loc }}^{p}$ becomes irrelevant.

We now turn to $L^{p} \rightarrow L^{q}$ estimates. Let $\overline{\mathcal{B}}(P)$ be the closed convex hull of the points in $\{(u, v)): 0 \leq u \leq 1, v \geq u\}$ and the points

$$
\left.\left(\frac{\mu+1}{\mu+\nu+1}, \frac{\mu}{\mu+\nu+1}\right), \quad(\mu, \nu) \in \tau(P)\right)
$$

\footnotetext{
${ }^{1}$ In [3], Christ, Nagel, Stein and Wainger independently introduced a curvature condition in terms of the subspaces $T_{P}^{1,0} \mathcal{M}, T_{P}^{0,1} \mathcal{M}$ and proved the equivalence with their original curvature assumption.
} 
Let $\underline{\mathcal{B}}(P)$ be the closed convex hull of the points in $\{(u, v)): 0 \leq u \leq 1, v \geq u\}$ and the points

$$
\left.\left(\frac{\frac{\mu}{n_{L}-1}+1}{\frac{\mu}{n_{L}-1}+\frac{\nu}{n_{R}-1}+1}, \frac{\frac{\mu}{n_{L}-1}}{\frac{\mu}{n_{L}-1}+\frac{\nu}{n_{R}-1}+1}\right), \quad(\mu, \nu) \in \tau(P)\right) .
$$

Again

$$
\overline{\mathcal{B}}(P)=\underline{\mathcal{B}}(P) \quad \text { if } n_{L}=n_{R}=2 .
$$

Theorem 1.2. Suppose that $\operatorname{codim} \mathcal{M}=1, P \in \mathcal{M}$ and $1 \leq p \leq \infty$.

(i) There is a neighborhood $\mathcal{U}$ of $P$ such that if $\operatorname{supp} \chi \subset \overline{\mathcal{U}}$ and if $(1 / p, 1, q)$ belongs to the interior of $\overline{\mathcal{B}}(P)$, then $R$ is bounded from $L_{\text {comp }}^{p}(\mathfrak{Y})$ into $L_{\text {loc }}^{q}(\mathfrak{X})$.

(ii) Suppose $\operatorname{dim} \mathfrak{X}=\operatorname{dim} \mathfrak{Y}=2$ and that $R$ is bounded from $L_{\text {comp }}^{p}(\mathfrak{Y})$ into $L_{\text {loc }}^{q}(\mathfrak{X})$. If $\chi(P) \neq 0$, then $(1 / p, 1 / q)$ belongs to $\underline{\mathcal{B}}(P)$.

Consider now the two-dimensional situation. If the principal symbol is multiplied by a suitable damping factor, then one can obtain the same $L^{2}$ Sobolev estimates as in the nondegenerate case, without assuming finite type conditions; see [21]. An essentially sharp version of this is

Theorem 1.3. Suppose $\operatorname{dim} \mathfrak{X}=\operatorname{dim} \mathfrak{Y}=2$ and $\operatorname{codim} \mathcal{M}=1$. Let $\mathcal{U}$ be an open set containing supp $\chi$ and let $X \in \mathcal{V}^{1,0}(\mathcal{U}), Y \in \mathcal{V}^{0,1}(\mathcal{U})$, nonvanishing in $\mathcal{U}$. Let $\omega$ be a section in $T^{*, \perp} \mathcal{M}$, nonvanishing over $\mathcal{U}$, and let $J_{\gamma}(x, y)=\left|\langle\omega,[X, Y]\rangle_{(x, y)}\right|^{\gamma}$. Define

$$
\mathcal{R}_{\gamma} f(x)=\int_{\mathcal{M}_{x}} \chi(x, y) J_{\gamma}(x, y) f(y) d \sigma_{x}(y) .
$$

If $\gamma>1 / 2$, then $\mathcal{R}_{\gamma}$ maps $L_{\text {comp }}^{2}$ boundedly to $L_{1 / 2, \text { loc }}^{2}$.

Remarks. We state some immediate consequences and further comments; here $\operatorname{codim} \mathcal{M}=1$ and in fact $\operatorname{dim} \mathfrak{X}=\operatorname{dim} \mathfrak{Y}=2$, unless otherwise stated.

(1) If $n=n(P)=\min \{|\mu|+|\nu|:(\mu, \nu) \in \tau(P)\}$ is the Hörmander type of $\mathcal{M}$ at $P$, then $\overline{\mathcal{A}}(P)$ and $\overline{\mathcal{B}}(P)$ are polygonal regions with at most $n+1$ extreme points; see Lemma 5.3 below. The operator $R$ is bounded from $L_{\text {comp }}^{p}$ to $L_{\alpha, \text { loc }}^{p}$ if $(\alpha, 1 / p) \in \bigcap_{P \in \operatorname{supp} \chi} \operatorname{Int}(\overline{\mathcal{A}}(P))$, and this result is essentially sharp, up to endpoints.

(2) Suppose that $\mu \geq 1$ and $\nu \geq 1$ and suppose that $\mathcal{M}$ is of type $\preceq(\mu, \nu)$ at every $P \in \operatorname{supp} \chi$. Let $p=1+\nu \mu^{-1}$ and $\alpha<(\mu+\nu)^{-1}$. Then $R$ is bounded from $L_{s}^{p}$ into $L_{s+\alpha}^{p}$, moreover $R$ is bounded from $L^{\frac{\mu+\nu+1}{\mu+1}}$ to $L^{\frac{\mu+\nu+1}{\mu}-\varepsilon}$. The first statement implies the second and then also the positive results of Theorems 1.1 and 1.2 , by multiple interpolation.

(3) Suppose that $R$ maps $L^{p}$ to $L_{\alpha}^{p}$ and that $\chi(P) \neq 0$. Then necessarily $(1-\alpha)^{-1} \leq p \leq \alpha^{-1}$; moreover there is a pair $(\mu, \nu)$ of positive integers with $\mu+\nu \leq \alpha^{-1}$, so that $\mathcal{M}$ is of type $(\mu, \nu)$ at $P$. Thus the reciprocal of the Hörmander type is the maximal gain of smoothness which can occur in some $L^{p}$ space.

(4) Suppose that $1 \leq p<q \leq \infty$ and $R$ maps $L^{p}$ to $L^{q}$. Suppose also that $\chi(P) \neq 0$. Then $\mathcal{M}$ is of type $(\mu, \nu)$ at $\left(x_{0}, y_{0}\right)$ for some pair $(\mu, \nu)$ of positive integers with $|\mu|+|\nu|+1 \leq(1 / p-1 / q)^{-1}$.

(5) By (3) and (4) the regions $\overline{\mathcal{A}}(P)$ and $\overline{\mathcal{B}}(P)$ have at least three extreme points if and only if $\mathcal{M}$ is of finite type at $P$. They are triangles in the model case in $\mathbb{R}^{2}$ where $\mathcal{M}$ is given by $y_{2}=x_{2}+x_{1}^{\mu} y_{1}^{\nu}$ and $P=(0,0)$. Here $\mathcal{M}$ is of type $(\mu, \nu)$ at $(0,0)$. Also note that $\mathcal{M}$ is of type $(1, \nu)$ at all points with $x_{1} \neq 0, y_{1}=0$, of 
type $(\mu, 1)$ for all points with $x_{1}=0, y_{1} \neq 0$, and of type $(1,1)$ for all points with $x_{1} \neq 0, y_{1} \neq 0$.

(6) Let $n=2 k$. The region $\overline{\mathcal{A}}((0,0))$ has $n+1$ extreme points if $\mathcal{M} \subset \mathbb{R}^{2} \times \mathbb{R}^{2}$ is given by $y_{2}=x_{2}+x_{1}^{k} y_{1}^{k}+\sum_{j=1}^{k-1}\left(x_{1}^{j} y_{1}^{2^{k-j} k}+x_{1}^{2^{k-j} k} y_{1}^{j}\right)$. If $n=2 k+1$, then an appropriate model example is given by $y_{2}=x_{2}+\sum_{j=1}^{k-1}\left(x_{1}^{j} y_{1}^{2^{k-j-1} k}+x_{1}^{2^{k-j-1}} k y_{1}^{j}\right)$.

(7) Theorem 1.1 is an extension of results in [19] since $\mathcal{M}$ satisfies a left finite type condition of order $m$, in the sense of [19], if and only if $\mathcal{M}$ is of type $\preceq(1, m-1)$; and $\mathcal{M}$ satisfies a right finite type condition of order $m$ if $\mathcal{M}$ is of type $\preceq(m-1,1)$. Cf. Corollary 3.7 below.

(8) It would be interesting to formulate the type $(\mu, \nu)$ condition for exponentials of vector fields $\gamma(x, t) \sim \exp \left(t^{\alpha} U_{\alpha} / \alpha !\right)$ as considered in [3]. For such conditions in the case where $\pi_{L}$ or $\pi_{R}$ are Whitney folds, see [15]. For similar computations in the presence of cusp singularities, see [5].

(9) Our theorems rely on a crucial $L^{2}$ estimate for Radon transforms proved in [19]; see (5.3) below. In fact Theorem 1.3 is an almost immediate consequence of this estimate. We note that an erroneous argument in [19] is corrected in the appendix, $\S 7$.

(10) An approach different from the one in this paper was previously used by Greenleaf and the author ([4], [5]) to obtain endpoint $L^{2}$ estimates for Fourier integral operators in specific situations (one sided folds and simple cusps). We plan to extend the methods of [19] to obtain $L^{2}$ estimates for Radon transforms associated with submanifolds of higher codimension, assuming finite type conditions.

(11) At this time it seems open in exactly which cases one can obtain sharp endpoint results, even in two dimensions. The case $\mu+\nu=3$ is completely understood (see [12], [15], [13], [19], [20], [4]). Some endpoint estimates for operators of type $(1, \nu)$ were obtained by the author in [19]; these operators map $L_{s}^{p}$ to $L_{s+1 / p}^{p}$ if $p>\nu+1$ and $L^{p}$ to $L^{2 p}$ if $p>(\nu+2) / 2$; moreover by duality operators of type $(\mu, 1) \operatorname{map} L_{s}^{p}$ to $L_{s+1 / p^{\prime}}^{p}$ if $p<(\mu+1) / \mu$ and $L^{p}$ to $L^{p /(2-p)}$ if $p \leq(\mu+2) /(\mu+1)$. Phong and Stein [16] established sharp $L^{p}$ endpoint results for a number of interesting examples, namely when $\mathcal{M}$ is given by $y_{2}=x_{2}+h\left(x_{1}, y_{1}\right)$ where $h$ is a homogeneous polynomial of degree $n, h\left(x_{1}, y_{1}\right)=\sum_{j=0}^{n} a_{j} x_{1}^{j} y_{1}^{n-j}$. If $a_{k} \neq 0$ and $1 \leq k \leq n-1$, then $\mathcal{M}$ is of type $(k, n-k)$ at $P=(0,0)$. Recently they obtained sharp $L^{2}$ results for more general semi-translation invariant cases, namely when $h\left(x_{1}, y_{1}\right)$ is assumed to be real analytic; see [17]. At least one endpoint estimate is known to fail even in the translation invariant case; for $h\left(x_{1}, y_{1}\right)=\left(x_{1}-y_{1}\right)^{n}$, M. Christ [2] showed that the corresponding averaging operator $R$ does not map $L_{s}^{n}$ to $L_{s+1 / n}^{n}$. Observe that in this case $\mathcal{M}$ is of type $(\mu, \nu)$, whenever $\mu+\nu=n$, and it is also known that $R$ maps $L_{s}^{p}$ to $L_{s+1 / n}^{p}$ if $n /(n-1)<p<n$; cf. [19], [16] and [2].

(12) Consider the special case where $\mathcal{M}$ is given by $y_{2}=x_{2}+h\left(x_{1}, y_{1}\right)$. Using suitable cutoff functions $\chi$ one can use Theorem 1.3 and Plancherel's theorem to derive an estimate for the damped oscillatory integral operator acting on functions on the real line, namely

$$
T_{\lambda, \gamma} g(v)=\int e^{i \lambda h(v, w)} g(w)\left|h_{v w}^{\prime \prime}(v, w)\right|^{\gamma} \eta(v, w) d w ;
$$

here $\eta \in C_{0}^{\infty}(\mathbb{R} \times \mathbb{R})$. The result is that for $\lambda \geq 1$ the $L^{2}$ norm of $T_{\lambda, \gamma}$ is $O\left(|\lambda|^{-1 / 2}\right)$ if $\gamma>1 / 2$. We learned at the harmonic analysis conference in Varenna, April 1997, where this work was presented, that Phong and Stein have recently obtained 
the bound $O\left(|\lambda|^{-1 / 2}\right)$ even for $\gamma=1 / 2$, provided that $h$ is real analytic; for some previously considered model cases see [16]. It would be interesting to extend the endpoint result to classes of oscillatory integral operators and operators of Radon transform type satisfying finite type or suitable convexity assumptions, without the hypothesis of real analyticity.

In $\S 2$ we review the condition of type $(1,1)$ and in $\S 3$ we give some convenient formulation of the finite type condition for hypersurfaces. $\S 4$ contains an elementary estimate for integral operators which relies on the finite type condition. In $\S 5$ we combine this with $L^{2}$ estimates from [19] to derive the $L^{p}$ Sobolev estimates of Theorem 1.1. Necessary conditions are discussed in $\S 6$.

\section{Preliminary REMARKS}

In this section we consider coordinate patches on $\mathfrak{X}$ and $\mathfrak{Y}$; we may assume that $\mathfrak{X}$ and $\mathfrak{Y}$ are itself open subsets of $\mathbb{R}^{n_{L}}$ and $\mathbb{R}^{n_{R}}$, respectively.

We assume that $\operatorname{codim} \mathcal{M}=\ell$ and that $\mathcal{M}$ is described by an $\mathbb{R}^{\ell}$-valued defining function,

$$
\mathcal{M}=\{(x, y): \Phi(x, y)=0\} .
$$

Here $\Phi=\left(\Phi^{1}, \ldots, \Phi^{\ell}\right)$ and

$$
\operatorname{rank} \Phi_{x}^{\prime}=\operatorname{rank} \Phi_{y}^{\prime}=\ell
$$

Recall that the $L^{2}$ regularity properties are determined by the projections of $\mathcal{C}=\left(N^{*} \mathcal{M}\right)^{\prime} \backslash 0$ to $T^{*} \mathfrak{X}$ and $T^{*} \mathfrak{Y}$. Writing $R$ as the Fourier integral operator with frequency variable $\theta \in \mathbb{R}^{\ell}$ we see that $\mathcal{C}$ is parametrized in the usual way as

$$
\left\{\left(x, d_{x} \Psi, y,-d_{y} \Psi\right): d_{\theta} \Psi=0\right\},
$$

with the phase function $\Psi(x, y, \theta)=\langle\theta, \Phi(x, y)\rangle=\sum_{i=1}^{\ell} \theta_{i} \Phi^{i}(x, y)$.

The situation of maximal nondegeneracy occurs if differentials of the projections $\pi_{L}$ and $\pi_{R}$ have maximal rank; that is, if the rank of the $\left(n_{L}+\ell\right) \times\left(n_{R}+\ell\right)$ matrix

$$
\mathcal{J}_{\Phi}(x, y, \theta)=\left(\begin{array}{ll}
\Psi_{x y}^{\prime \prime} & \Psi_{x \theta}^{\prime \prime} \\
\Psi_{\theta y}^{\prime \prime} & \Psi_{\theta \theta}^{\prime \prime}
\end{array}\right)=\left(\begin{array}{cc}
\langle\theta, \Phi\rangle_{x y}^{\prime \prime} & \Phi_{x}^{\prime} \\
\Phi_{y}^{\prime} & 0
\end{array}\right)
$$

is maximal, for all $\theta \neq 0$. In particular the twisted normal bundle $\mathcal{C}=\left(N^{*} \mathcal{M}\right)^{\prime}$ is a local canonical graph near $P$ if $n_{L}=n_{R}$ and $\operatorname{det}\left(\mathcal{J}_{\Phi}\right) \neq 0$ for all $\theta \neq 0$.

It will be useful to reformulate the finite type condition in terms of the canonical relation $\mathcal{C}$ and the matrix $\mathcal{J}_{\Phi}$. We shall need the behavior of $\mathcal{J}_{\Phi}$ under changes of variables in $\mathfrak{X}$ and $\mathfrak{Y}$. Let $\psi_{L}$ and $\psi_{R}$ denote diffeomorphisms in $\mathfrak{X}$ and $\mathfrak{Y}$, respectively, and define $\psi(w, z)=\left(\psi_{L}(w), \psi_{R}(z)\right)$. Then

$$
\mathcal{J}_{\Phi \circ \psi}(w, z, \theta)=\left(\begin{array}{cc}
D \psi_{L}{ }^{t} & 0 \\
0 & I
\end{array}\right)\left(\begin{array}{cc}
\langle\theta, \Phi\rangle_{x y}^{\prime \prime} & \Phi_{x}^{\prime t} \\
\Phi_{y}^{\prime} & 0
\end{array}\right)\left(\begin{array}{cc}
D \psi_{R} & 0 \\
0 & I
\end{array}\right)
$$

where the derivatives of $\Phi$ are evaluated at $(x, y)=\left(\psi_{L}(w), \psi_{R}(z)\right)$.

In order to reformulate the finite type condition in terms of $\mathcal{J}_{\Phi}$ we consider vector fields $X=\sum_{j} a_{j}(x, y) \partial / \partial x_{j}$ of type $(1,0)$ and vector fields $Y=\sum_{k} b_{k}(x, y) \partial / \partial y_{k}$ of type $(0,1)$ and extend them to smooth vector fields in a neighborhood of $\mathcal{M}$. 
For these vector fields we define the bilinear form

$$
\begin{aligned}
B_{\theta}(X, Y)_{P} & =\left.a^{t}\langle\theta, \Phi\rangle_{x y}^{\prime \prime} b\right|_{P} \\
& =\left.\left(\begin{array}{ll}
a^{t} & 0
\end{array}\right)\left(\begin{array}{cc}
\langle\theta, \Phi\rangle_{x y}^{\prime \prime} & \Phi_{x}^{\prime t} \\
\Phi_{y}^{\prime} & 0
\end{array}\right)\left(\begin{array}{l}
b \\
0
\end{array}\right)\right|_{P}
\end{aligned}
$$

which depends linearly on $\theta$. (2.3) shows the invariance of $B_{\theta}$, namely

$$
\left(D \psi_{L} a\right)^{t}\langle\theta, \Phi \circ \psi\rangle_{w z}^{\prime \prime}\left(D \psi_{R} b\right)=\left.a^{t}\langle\theta, \Phi\rangle_{x y}^{\prime \prime} b\right|_{(x, y)=\left(\psi_{L}(w), \psi_{R}(z)\right)} .
$$

Note also the behavior of this bilinear form under changes in the defining function. If $(x, y) \mapsto A(x, y)$ is smooth with values in the general linear group $G L\left(\mathbb{R}^{\ell}\right)$, and if $\widetilde{\Phi}(x, y)=A(x, y) \Phi(x, y), \widetilde{\theta}=\left(A^{t}(x, y)\right)^{-1} \theta$, then

$$
a^{t}\langle\widetilde{\theta}, \widetilde{\Phi}\rangle_{x y}^{\prime \prime} b=a^{t}\langle\theta, \Phi\rangle_{x y}^{\prime \prime} b .
$$

On $\mathfrak{X} \times \mathfrak{Y}$ we have exterior derivatives $d_{L}$ and $d_{R}$ defined, as natural extensions to $\mathfrak{X} \times \mathfrak{Y}$ of $d_{x}$ on $\mathfrak{X}$ and $d_{y}$ on $\mathfrak{Y}$, by $d_{L} \omega_{(x, y)}=d_{x}\left(\omega_{(\cdot, y)}\right)_{x}$ and $d_{R} \omega_{(x, y)}=d_{y}\left(\omega_{(x, \cdot)}\right)_{y}$. Now consider the forms

$$
d_{L} \Phi^{i}-d_{R} \Phi^{i}, \quad i=1, \ldots, \ell,
$$

on $T^{*}(\mathfrak{X} \times \mathfrak{Y})$, which span the fiber of the canonical relation $\left(N^{*} \mathcal{M}\right)^{\prime}$ at $P$. There are the corresponding forms $d_{L} \Phi^{i}+d_{R} \Phi^{i}$ that span the fiber of $N^{*} \mathcal{M}$ at $P$ and in view of the linear independence of $\left\{d_{L} \Phi_{P}^{i}, d_{R} \Phi_{P}^{i} ; i=1, \ldots, \ell\right\}$ the set $\left\{d_{L} \Phi^{i}-\right.$ $\left.d_{R} \Phi^{i}, d_{L} \Phi^{i}+d_{R} \Phi^{i} ; i=1, \ldots, \ell\right\}$ is linearly independent at $P$. We restrict the forms in (2.7) to linear forms acting on tangent vectors in $T_{P} \mathcal{M}$;

$$
\omega^{i}=\imath^{*}\left(d_{L} \Phi^{i}-d_{R} \Phi^{i}\right)
$$

where $\imath^{*}$ is the pullback map of the inclusion $\imath: \mathcal{M} \rightarrow \mathfrak{X} \times \mathfrak{Y}$. Then the $\omega_{P}^{i}$ are linearly independent in $T_{P}^{*} \mathcal{M}$, and since they annihilate vectors of type $(1,0)$ and $(0,1)$, they form a basis of $T_{P}^{*, \perp} \mathcal{M}$.

Lemma 2.1. Suppose that $\omega_{\theta}=\sum_{i=1}^{\ell} \theta_{i} \omega^{i}$, with $\omega^{i}$ as in (2.8)-(2.9). Let $X \in$ $\mathcal{V}^{1,0}(P)$ and $Y \in \mathcal{V}^{0,1}(P)$. Then

$$
\left\langle\omega_{\theta},[X, Y]\right\rangle=2 B_{\theta}(X, Y) .
$$

Proof. If $X=\sum_{j} a_{j} \partial / \partial x_{j}$ and $Y=\sum_{k} b_{k} \partial / \partial y_{k}$, then $[X, Y]=\sum_{j} v_{j} \partial / \partial x_{j}+$ $\sum_{k} w_{k} \partial / \partial y_{k}$ with $v=-\left(D_{y} a\right) b$ and $w=\left(D_{x} b\right) a$. Since $\Phi_{x}^{\prime} a=0$ and $\Phi_{y}^{\prime} b=0$ we obtain by differentiation

$$
\left\langle\theta, \Phi_{x}^{\prime}\right\rangle\left(D_{y} a\right) b=-a^{t}\langle\theta, \Phi\rangle_{x y}^{\prime \prime} b=\left\langle\theta, \Phi_{y}^{\prime}\right\rangle\left(D_{x} b\right) a .
$$

By (2.8)

$$
\begin{aligned}
\left\langle\omega_{\theta},[X, Y]\right\rangle & =\sum_{i=1}^{\ell} \theta_{i}\left\langle d_{L} \Phi^{i}-d_{R} \Phi^{i},[X, Y]\right\rangle \\
& =-\left\langle\theta, \Phi_{x}^{\prime}\right\rangle\left(D_{y} a\right) b-\left\langle\theta, \Phi_{y}^{\prime}\right\rangle\left(D_{x} b\right) a \\
& =2 a^{t}\langle\theta, \Phi\rangle_{x y}^{\prime \prime} b
\end{aligned}
$$

where all the expressions are evaluated at $P \in \mathcal{M}$. 
Remarks. (i) Let $(P, \lambda) \in T^{*, \perp} \mathcal{M}, \lambda=\sum_{i=1}^{\ell} \omega_{P}^{i}$. Then $T^{*} \mathcal{M}$ is of type $(1,1)$ at $(P, \lambda)$ if and only if $B_{\theta}(X, Y)_{P} \neq 0$ for some $X \in \mathcal{V}^{1,0}(P), Y \in \mathcal{V}^{0,1}(P)$.

(ii) An alternative description of $B_{\theta}$ can be obtained using differential forms. Note that $d_{L} d_{R} \Phi^{i}=\sum_{j} \sum_{k} \frac{\partial^{2} \Phi^{i}}{\partial x_{j} \partial y_{k}} d x_{j} \wedge d y_{k}$ so that for $X \in \mathcal{V}^{1,0}(P), Y \in \mathcal{V}^{0,1}(P)$

$$
\left\langle d_{L} d_{R} \theta \cdot \Phi \mid X \wedge Y\right\rangle=B_{\theta}(X, Y) ;
$$

here we use the standard inner product $\langle\cdot \mid \cdot\rangle$ for $p$-forms, with the normalization that for $p$-vectors $x=x_{1} \wedge \cdots \wedge x_{p}$ and $p$-forms $\lambda=\lambda_{1} \wedge \cdots \wedge \lambda_{p}$ we have $\langle\lambda \mid x\rangle=$ $\operatorname{det}\left(\left\langle\lambda_{i}, x_{j}\right\rangle\right)$. Following Kohn [11] the result of Lemma 2.1 can be derived using a standard formula for the exterior derivative of a 1-form $\omega$, namely $\langle d \omega \mid X \wedge Y\rangle=$ $X(\langle\omega \mid Y\rangle)-Y(\langle\omega \mid X\rangle)-\langle\omega \mid[X, Y]\rangle$; see [23, p. 103].

Let $X \in \mathcal{V}^{1,0}(P)$ and $Y \in \mathcal{V}^{0,1}(P)$. Then $\left\langle d_{L} \Phi^{i} \mid X\right\rangle=0,\left\langle d_{R} \Phi^{i} \mid X\right\rangle=0$, $\left\langle d_{R} \Phi^{i} \mid Y\right\rangle=0$, and $\left\langle d_{L} \Phi^{i} \mid Y\right\rangle=0$. Now $d d=d_{L} d_{L}=d_{R} d_{R}=0$ and therefore $d_{R} d_{L}=-d_{L} d_{R}$. We compute $\left\langle d_{L} d_{R} \Phi^{i} \mid X \wedge Y\right\rangle=\left\langle d d_{R} \Phi^{i} \mid X \wedge Y\right\rangle=-\left\langle d_{R} \Phi^{i} \mid[X, Y]\right\rangle$ and $\left\langle d_{L} d_{R} \Phi^{i} \mid X \wedge Y\right\rangle=-\left\langle d d_{L} \Phi^{i} \mid X \wedge Y\right\rangle=\left\langle d_{L} \Phi^{i} \mid[X, Y]\right\rangle$. If $e_{1}, \ldots, e_{\ell}$ denote the standard unit vectors in $\mathbb{R}^{\ell}$ we see that on $\mathcal{M}$

$$
2 B_{e_{i}}(X, Y)=2\left\langle d_{L} d_{R} \Phi^{i} \mid X \wedge Y\right\rangle=\left\langle d_{L} \Phi^{i}-d_{R} \Phi^{i} \mid[X, Y]\right\rangle=\left\langle\omega^{i} \mid[X, Y]\right\rangle
$$

which is equivalent to the statement of Lemma 2.1 .

(iii) There is a formal analogy with the Levi form in several complex variables, which is apparent from rewriting (2.9) as

$$
2 B_{\theta}(X, Y)=\frac{1}{2 i}\left\langle\omega_{\theta},[X-i Y, X+i Y]\right\rangle
$$

see also the discussion in [14, p. 114].

Lemma 2.2. Let $T_{1}, \ldots, T_{\ell}$ be vector fields on $\mathcal{M}$ satisfying

$$
\left\langle\omega^{i}, T_{j}\right\rangle= \begin{cases}1 & \text { if } i=j, \\ 0 & \text { if } i \neq j .\end{cases}
$$

Let $X_{1}, \ldots, X_{n_{L}-\ell} \in \mathcal{V}^{1,0}(P), Y_{1}, \ldots, Y_{n_{R}} \in \mathcal{V}^{0,1}(P)$ so that $\left\{X_{i}, Y_{j}, T_{k}\right\}_{P}$ form a basis of $T_{P}^{*} \mathcal{M}$. Let $\left\{e_{1}, \ldots, e_{\ell}\right\}$ be the standard basis of $\mathbb{R}^{\ell}$. Then there are $C^{\infty}$ functions $\alpha_{i}, \beta_{j}$ so that

$$
[X, Y]=\sum_{j=1}^{n_{L}-\ell} \alpha_{j} X_{j}+\sum_{k=1}^{n_{R}-\ell} \beta_{k} Y_{k}+\sum_{s=1}^{\ell} B_{e_{s}}(X, Y) T_{s} .
$$

Proof. This is an immediate consequence of Lemma 2.1.

It will also be convenient to note the following formula in the particular case where the manifolds $\mathcal{M}_{x}, \mathcal{M}^{y}$ are curves.

Lemma 2.3. Suppose $\operatorname{dim} \mathfrak{X}=\operatorname{dim} \mathfrak{Y}=d, \operatorname{codim} \mathcal{M}=d-1, P=(x, y) \in \mathcal{M}$. Let $\omega_{\theta}=\sum_{i=1}^{l} \theta_{i} \omega^{i}$, where $\omega^{i}$ is as in (2.8). Let $\Gamma_{L}^{j}(x, y)$ be the $(d-1) \times(d-1)$ matrix obtained from the $(d-1) \times d$ matrix $\Phi_{x}^{\prime}$ by omitting the $j^{\text {th }}$ column, and let $\Gamma_{R}^{j}(x, y)$ be the $(d-1) \times(d-1)$ matrix obtained from $\left(B_{i j}\right)=\Phi_{y}^{\prime}$ by omitting the $j^{\text {th }}$ column. Let

$$
X=\sum_{j=1}^{d} a_{j}(x, y) \frac{\partial}{\partial x_{j}}, \quad Y=\sum_{k=1}^{d} b_{k}(x, y) \frac{\partial}{\partial y_{k}}
$$


where

$$
a_{j}(x, y)=(-1)^{j-1} \operatorname{det} \Gamma_{L}^{j}(x, y), \quad b_{k}(x, y)=(-1)^{k-1} \operatorname{det} \Gamma_{R}^{k}(x, y) .
$$

Then $X$ and $Y$ are vector fields of type $(1,0)$ and $(0,1)$, respectively, and

$$
(-1)^{d-1}\left\langle\omega_{\theta},[X, Y]\right\rangle=2 \frac{|a|^{2}|b|^{2}}{\operatorname{det} \Phi_{x}^{\prime} \Phi_{x}^{\prime t} \operatorname{det} \Phi_{y}^{\prime} \Phi_{y}^{\prime t}} \operatorname{det} \mathcal{J}_{\Phi}(\cdot, \cdot, \theta)
$$

In particular, if $d=2$, then $\left\langle\omega_{\theta},[X, Y]_{P}\right\rangle=-2 \operatorname{det} \mathcal{J}_{\Phi}(x, y, \theta)$.

Proof. Let $A=\Phi_{x}^{\prime}, B=\Phi_{y}^{\prime}, M=\langle\theta, \Phi\rangle_{x y}^{\prime \prime}$. Then $A a=B b=0$. Setting $\mathcal{A}^{t}=$ $\left(a, A^{t}\right)$ and $\mathcal{B}^{t}=\left(b, B^{t}\right)$ we have the identity

$$
\left(\begin{array}{cc}
\mathcal{A} & 0 \\
0 & I
\end{array}\right)\left(\begin{array}{cc}
M & A^{t} \\
B & 0
\end{array}\right)\left(\begin{array}{cc}
\mathcal{B}^{t} & 0 \\
0 & I
\end{array}\right)=\left(\begin{array}{ccc}
a^{t} M b & a^{t} M B^{t} & 0 \\
A M b & A M B^{t} & A A^{t} \\
0 & B B^{t} & 0
\end{array}\right) .
$$

A cofactor expansion yields that $\operatorname{det} \mathcal{A}=|a|^{2}$, $\operatorname{det} \mathcal{B}=|b|^{2}$. Therefore taking determinants we see that

$$
|a|^{2}|b|^{2} \operatorname{det} \mathcal{J}_{\Phi}=(-1)^{d-1} a^{t}\langle\theta, \Phi\rangle_{x y}^{\prime \prime} b \operatorname{det} \Phi_{x}^{\prime} \Phi_{x}^{\prime t} \operatorname{det} \Phi_{y} \Phi_{y}^{\prime t} .
$$

The asserted formula follows immediately from Lemma 2.1.

\section{Finite TYPE CONDITIONS FOR HYPERSURFACES}

In this section we assume that $\mathcal{M}$ is a hypersurface in $\mathfrak{X} \times \mathfrak{Y}$, given by the equation $\Phi(x, y)=0$. We shall need the notion of a differential operator of type $\preceq(\mu, \nu)$. The operator of multiplication by a function is said to be of type $(0,0)$. We say that $X$ is of type $(1,0)$ if $X=\sum_{j} a_{j} \partial / \partial x_{j}$ is a vector field, whose restriction of $X$ to $\mathcal{M}$ is tangential to both $\mathcal{M}$ and $\mathfrak{X}$. We say that $Y$ is of type $(0,1)$ if $Y=\sum_{j} b_{j} \partial / \partial y_{j}$ is a vector field whose restriction of $Y$ to $\mathcal{M}$ is tangential to $\mathcal{M}$ and $\mathfrak{Y}$. Let $\mu \geq 1, \nu \geq 1$; then the operator $L$ is defined to be of type $\preceq(\mu, \nu)$ if $L$ is of order $\leq \mu+\nu$ and if $L$ is a linear combination over $C^{\infty}$ of operators of order $(0,0)$ and differential operators of the form $g V_{1} \ldots V_{n}$ where each $V_{i}$ is of type $(1,0)$ or $(0,1)$, but at most $\mu$ of the $V_{i}$ are of type $(1,0)$ and at most $\nu$ of the $V_{i}$ are of type $(0,1)$. The $C^{\infty}$ module of operators of type $\preceq(\mu, \nu)$ in an open set $\mathcal{U}$ is denoted by $\mathfrak{L}^{\mu, \nu}(\mathcal{U})$, similarly we may define germs of differential operators and use the notation $\mathfrak{L}^{\mu, \nu}(P)$.

We set

$$
B(X, Y)=\left.B_{\theta}(X, Y)\right|_{\theta=1}, \quad \omega=\left.\omega_{\theta}\right|_{\theta=1} .
$$

We shall need to work with tangential vector fields $T$ satisfying $\langle\omega, T\rangle_{P}=1$; a natural choice is

$$
T=\frac{1}{2}\left(\frac{1}{\left|\Phi_{x}^{\prime}\right|^{2}} \sum_{j=1}^{n_{L}} \frac{\partial \Phi}{\partial x_{j}} \frac{\partial}{\partial x_{j}}-\frac{1}{\left|\Phi_{y}^{\prime}\right|^{2}} \sum_{k=1}^{n_{R}} \frac{\partial \Phi}{\partial y_{k}} \frac{\partial}{\partial y_{k}}\right) .
$$

Theorem 3.1. Suppose $P=(x, y) \in \mathcal{M}$. The following conditions are equivalent.

(i) $\mathcal{M}$ is of type $(\mu, \nu)$ at $P$.

(ii) There are $\mu$ vector fields $V_{1}, \ldots, V_{\mu} \in \mathcal{V}^{1,0}(P)$ and $\nu$ vector fields $V_{\mu+1}, \ldots$, $V_{\mu+\nu} \in \mathcal{V}^{0,1}(P)$ so that

$$
\left\langle\omega, \operatorname{ad} V_{1} \cdots \operatorname{ad} V_{\mu+\nu-1}\left(V_{\mu+\nu}\right)\right\rangle_{P} \neq 0
$$

but $\langle\omega, V\rangle_{P}=0$ for any vector field $V$ of type $\prec(\mu, \nu)$. 
(iii) There are $\mu$ vector fields $V_{1}, \ldots, V_{\mu} \in \mathcal{V}^{1,0}(P)$ and $\nu$ vector fields $V_{\mu+1}, \ldots$, $V_{\mu+\nu} \in \mathcal{V}^{0,1}(P)$ so that the following holds. For any choice of $C^{\infty}$ functions $h_{i}$ with $h_{i}(P) \neq 0$, for any permutation $\pi$ of $\{1, \ldots, \mu+\nu\}$ and for $W_{i}:=h_{i} V_{\pi(i)}$

$$
\left\langle\omega, \operatorname{ad} W_{1} \ldots \operatorname{ad} W_{\mu+\nu-1}\left(W_{\mu+\nu}\right)\right\rangle_{P} \neq 0
$$

but $\langle\omega, V\rangle_{P}=0$ for any vector field $V$ of type $\prec(\mu, \nu)$.

(iv) There are vector fields $X \in \mathcal{V}^{1,0}(P)$ and $Y \in \mathcal{V}^{0,1}(P)$, and a differential operator $L$ of type $\preceq(\mu-1, \nu-1)$ so that $L[B(X, Y)] \neq 0$, but $\widetilde{L}[B(\widetilde{X}, \widetilde{Y})]=0$ for all differential operators $\widetilde{L}$ of type $\prec(\mu-1, \nu-1)$ and all $\widetilde{X} \in \mathcal{V}^{1,0}(P), \widetilde{Y} \in \mathcal{V}^{0,1}(P)$.

A stronger variant of this theorem is obtained in Theorem 3.6 below.

We shall deduce Theorem 3.1 by induction over $n=\mu+\nu$ from various elementary lemmata.

Lemma 3.2. Let $V_{1}, \ldots, V_{n}$ be vector fields in $\mathcal{V}(P)$ and let $\widetilde{V}_{i}=f_{i} V_{i}$ where $f_{i} \in$ $C^{\infty}$. Then

$$
\widetilde{V}_{1} \widetilde{V}_{2} \cdots \widetilde{V}_{n}=f_{1} \cdots f_{n} V_{1} \cdots V_{n}+\sum_{\substack{\emptyset \neq A \\ \subset\{1, \ldots, n\}}} \sum_{\mathfrak{B}} L_{\mathfrak{B}}\left(f_{1}, \ldots, f_{n}\right) V_{A}
$$

here $V_{A}=V_{i_{1}} V_{i_{2}} \cdots V_{i_{n}}$ if $\left(i_{1}, \ldots, i_{n}\right) \in A$ and $i_{1}<\cdots<i_{n} . A^{c}$ denotes the complement of $A$ in $\{1, \ldots, n\}$ and $L_{\mathfrak{B}}\left(f_{1}, \ldots, f_{n}\right)$ is a finite sum of expressions of the form

$$
c \prod_{i \in B_{0}} f_{i} \prod_{k=1}^{N(\mathfrak{B})} V_{j_{1}^{k}} \cdots V_{j_{s(k)}^{k}} f_{k}
$$

where $\mathfrak{B}=\left\{B_{0}, \ldots, B_{N(\mathfrak{B})}\right\}$ is a partition of the set $A^{c}$ as a disjoint union, $A^{c}=$ $B_{0} \cup \cdots \cup B_{N(\mathfrak{B})}$, with $B_{k}=\left\{j_{1}^{k}, \ldots, j_{s(k)}^{k}\right\}$ and $j_{\nu}^{k}<k$.

Proof. This follows by a straightforward computation from repeated applications of the product rule.

For $j \geq 0, k \geq 0$ let $\mathcal{I}^{j, k}$ be the ideal in $\mathcal{V}(P)$ generated by vector fields of the form $L(B(X, Y)) W$, where $W \in \mathcal{V}(P)$ and $L \in \mathfrak{L}^{j, k}(P), X \in \mathcal{V}^{1,0}(P), Y \in \mathcal{V}^{0,1}(P)$. We set $\mathcal{I}^{-1,0}=\mathcal{I}^{0,-1}=\{0\}$.

Lemma 3.3. Suppose $n=\mu+\nu$ and suppose $V_{1}, \ldots, V_{n}$ are vector fields, $\mu$ of them in $\mathcal{V}^{1,0}(P)$, $\nu$ of them in $\mathcal{V}^{0,1}(P)$. Let $T \in \mathcal{V}(P)$ so that $\langle\omega, T\rangle=1$. Then

$$
\operatorname{ad} V_{1} \cdots \operatorname{ad} V_{n-1}\left(V_{n}\right)=2 V_{1} \cdots V_{n-2} B\left(V_{n-1}, V_{n}\right) T+W_{1}+W_{2}+Z_{1}+Z_{2}
$$

where $W_{1} \in \mathcal{V}^{\mu-1, \nu}(P), W_{2} \in \mathcal{V}^{\mu, \nu-1}(P), Z_{1} \in \mathcal{I}^{\mu-2, \nu-1}(P), Z_{2} \in \mathcal{I}^{\mu-1, \nu-2}(P)$.

Proof. This is easily shown by using the formula $\langle\omega,[X, Y]\rangle=2 B(X, Y)$ and induction.

The leading terms in (3.4) are not commutative in $V_{1}, \ldots, V_{n}$. The following Lemma shows that commutativity holds modulo suitable "negligible" terms.

Let $\mathfrak{J}_{j, k}^{n}(P)$ consist of all linear combinations of the expressions $g L_{1}\left[B_{k}(X, Y)\right] L_{2}$ where $X \in \mathcal{V}^{1,0}(P), Y \in \mathcal{V}^{0,1}(P), L_{1}$ is of type $\left(j^{\prime}, k^{\prime}\right)$ where $\left(j^{\prime}, k^{\prime}\right) \preceq(j, k)$ and $L_{2}$ is a differential operator so that $j^{\prime}+k^{\prime}+\operatorname{order}\left(L_{2}\right) \leq n$. 
Lemma 3.4. Let $V_{1}, \ldots, V_{n}$ be vector fields, $\mu$ of them in $\mathcal{V}^{1,0}(P)$, $\nu$ of them in $\mathcal{V}^{0,1}(P)$. Let $\pi, \pi^{\prime}$ be permutations of the set $\{1,2, \ldots, n\}$.

Then

$$
V_{\pi(1)} \cdots V_{\pi(n)}=V_{\pi^{\prime}(1)} \cdots V_{\pi^{\prime}(n)}+L_{1}+L_{2}+E
$$

here $L_{1} \in \mathfrak{L}^{\mu-1, \nu}, L_{2} \in \mathfrak{L}^{\mu, \nu-1}$; moreover $E \in \mathfrak{I}_{\mu-1, \nu-1}^{\mu+\nu-1}$.

Proof. It suffices to prove this theorem for $\pi^{\prime}$ being the identity. We note by Lemma 2.2 that

$$
\begin{aligned}
V_{1} \cdots V_{k-2} V_{k-1} V_{k} V_{k+1} \cdots V_{n} & =V_{1} \cdots V_{k-2} V_{k} V_{k-1} V_{k+1} \cdots V_{n} \\
& +V_{1} \cdots V_{k-2} X V_{k+1} \cdots V_{n} \\
& +V_{1} \cdots V_{k-1} Y V_{k+1} \cdots V_{n} \\
& +2 \sum_{k=1}^{\ell} V_{1} \cdots V_{k-2} \mathcal{B}_{k}\left(V_{k-1}, V_{k}\right) T V_{k+1} \cdots V_{n}
\end{aligned}
$$

where $X \in \mathcal{V}^{1,0}(P), Y \in \mathcal{V}^{0,1}(P), \mathcal{B}_{k}\left(V_{k-1}, V_{k}\right)=B_{e_{k}}\left(V_{k-1}, V_{k}\right)$ if $V_{k-1} \in \mathcal{V}^{1,0}(P)$, $V_{k} \in \mathcal{V}^{0,1}(P)$ and $\mathcal{B}_{k}\left(V_{k-1}, V_{k}\right)=-B_{e_{k}}\left(V_{k}, V_{k-1}\right)$ if $V_{k} \in \mathcal{V}^{1,0}(P), V_{k-1} \in \mathcal{V}^{0,1}(P)$; moreover $\mathcal{B}_{k}\left(V_{k-1}, V_{k}\right)=0$ if $V_{k-1}$ and $V_{k}$ are both in $\mathcal{V}^{1,0}(P)$ or both in $\mathcal{V}^{0,1}(P)$. (3.5) and an application of Lemma 3.2 imply the assertion for transpositions $\pi$. A repetition of this argument shows the assertion for the permutation $\pi$ with $\pi(1)=k$, $\pi(j)=j-1$ for $2 \leq j \leq k$ and $\pi(j)=j$ for $j>k$. From this the general case follows easily by induction. In the induction step one uses that for $X \in \mathcal{V}^{1,0}(P)$, $Y \in \mathcal{V}^{0,1}(P), L \in \mathfrak{L}^{\mu, \nu}(P), E \in \mathfrak{I}_{\mu-1, \nu-1}^{\mu+\nu-1}$ we have that $X L \in \mathfrak{L}^{\mu+1, \nu}(P), Y L \in$ $\mathfrak{L}^{\mu, \nu+1}(P), X E \in \mathfrak{I}_{\mu, \nu-1}^{\mu+\nu}$, and $Y E \in \mathfrak{I}_{\mu-1, \nu}^{\mu+\nu}$.

Proof of Theorem 3.1. (i) is equivalent with (ii) by the definition of type $(\mu, \nu)$. (ii) is equivalent with (iii) by Lemma 3.2 and Lemma 3.4. (iii) is equivalent with (iv) by Lemma 3.3.

We now wish to show that in statements (ii), (iii) of Theorem 3.1 it is possible to work with a single vector field of type $(1,0)$ and a single vector field of type $(0,1)$. Our calculations are facilitated by a particular choice of coordinates in $\mathfrak{X}$ and $\mathfrak{Y}$ vanishing at the point $P$.

We shall use the notation $u=x_{n_{L}}, v=y_{n_{R}}$ and $y^{\prime}=\left(y_{1}, \ldots, y_{n_{R}-1}\right), x^{\prime}=$ $\left(x_{1}, \ldots, x_{n_{L}-1}\right)$, so that $x=\left(x^{\prime}, u\right), y=\left(y^{\prime}, v\right)$. We may assume that near $P=$ $(0,0)$ the manifold is given by

$$
v=S\left(x, y^{\prime}\right) \Longleftrightarrow u=\mathfrak{S}\left(y, x^{\prime}\right) .
$$

By suitable choices of the coordinates in $\mathfrak{X}$ and in $\mathfrak{Y}$ we can assume that

$$
\begin{aligned}
& S(x, 0)=x_{n_{L}} \equiv u, \\
& \mathfrak{S}(y, 0)=y_{n_{R}} \equiv v .
\end{aligned}
$$

In particular it follows by implicit differentiation that $\partial_{y}^{\alpha} S(0,0)=0$ for all multiindices $\alpha \neq(0, \ldots, 0,1)$.

Set $\Phi(x, y)=y_{n_{L}}-S\left(x, y^{\prime}\right)$. We shall work with the vector fields

$$
X_{j}=-\Phi_{x_{n_{L}}}^{\prime} \frac{\partial}{\partial x_{j}}+\Phi_{x_{j}}^{\prime} \frac{\partial}{\partial x_{n_{L}}}=S_{u}^{\prime} \frac{\partial}{\partial x_{j}}-S_{x_{j}}^{\prime} \frac{\partial}{\partial u}
$$


and

$$
Y_{k}=\Phi_{y_{n_{R}}}^{\prime} \frac{\partial}{\partial y_{k}}-\Phi_{y_{k}}^{\prime} \frac{\partial}{\partial y_{n_{R}}}=\frac{\partial}{\partial y_{k}}+S_{y_{k}}^{\prime} \frac{\partial}{\partial v} ;
$$

then a short computation using (2.4) shows that for $P=\left(x, y^{\prime}, S\left(x, y^{\prime}\right)\right)$

$$
B\left(X_{j}, Y_{k}\right)_{P}=S_{u}^{\prime} S_{x_{j} y_{k}}^{\prime \prime}-\left.S_{x_{j}}^{\prime} S_{u y_{k}}^{\prime \prime}\right|_{\left(x, y^{\prime}\right)} .
$$

If

$$
X=\sum_{j=1}^{n_{L}-1} a_{j}(x, y) X_{j} ; \quad Y=\sum_{k=1}^{n_{R}-1} b_{k}(x, y) Y_{k}
$$

with smooth functions $a_{j}, b_{k}$ and $a=\left(a_{1}, \ldots, a_{n_{L}-1}\right), b=\left(b_{1}, \ldots, b_{n_{R}-1}\right)$, then

$$
\begin{aligned}
B(X, Y) & =S_{u}^{\prime}\left\langle\alpha, \nabla_{x^{\prime}}\right\rangle\left\langle\beta, \nabla_{y^{\prime}}\right\rangle S-\left.\left\langle\alpha, \nabla_{x^{\prime}} S\right\rangle\left\langle\beta, \nabla_{y^{\prime}} S_{u}\right\rangle\right|_{\substack{\alpha=a(x, y) \\
\beta=b(x, y)}} \\
& =\sum_{j=1}^{n_{L}-1} \sum_{k=1}^{n_{R}-1} a_{j}(x, y) b_{k}(x, y)\left[S_{u}^{\prime} S_{x_{j} y_{k}}^{\prime \prime}-S_{x_{j}}^{\prime} S_{u y_{k}}^{\prime \prime}\right]_{(x, y)} .
\end{aligned}
$$

Lemma 3.5. Suppose that $\mu \geq 1, \nu \geq 1$ and suppose that in a neighborhhod $\mathcal{U}$ of $P$ coordinates vanishing at $P$ are chosen so that $\mathcal{M}$ is given by (3.6), (3.7). Let $a^{l}: \mathcal{U} \rightarrow \mathbb{R}^{n_{L}-1}, b^{m}: \mathcal{U} \rightarrow \mathbb{R}^{n_{R}-1}$ be smooth functions, $l=1, \ldots, \mu, m=1, \ldots, \nu$. For each $l, m$ define $X^{l}=\sum_{j=1}^{n_{L}-1} a_{j}^{l}(x, y) X_{j}, Y^{m}=\sum_{k=1}^{n_{R}-1} b_{k}^{m}(x, y) Y_{k}$, where $X_{j}$ and $Y_{k}$ are as in (3.8), (3.9). Let $V_{1}=X^{1}, V_{2}=Y^{1}$ and, if $\mu+\nu>1$, let $V_{3}, \ldots, V_{\mu+\nu}$ be a permutation of the vector fields $X^{2}, \ldots, X^{\mu}, Y^{2}, \ldots, Y^{\nu}$. Let

$$
\mathcal{L}=V_{3} \cdots V_{\mu+\nu}
$$

if $(1,1) \prec(\mu, \nu)$ and let $\mathcal{L}$ be the identity if $(\mu, \nu) \preceq(1,1)$. For fixed $(x, y)$ let $L_{x y}$ be the constant coefficient differential operator

$$
L_{x y}=\left.\left\langle\alpha^{1}, \nabla_{x^{\prime}}\right\rangle \cdots\left\langle\alpha^{l}, \nabla_{x^{\prime}}\right\rangle\left\langle\beta^{1}, \nabla_{y^{\prime}}\right\rangle \cdots\left\langle\beta^{m}, \nabla_{y^{\prime}}\right\rangle\right|_{\substack{\alpha^{l}=a^{l}(x, y) \\ \beta^{m}=b^{m}(x, y)}} .
$$

Let $\mathcal{I}_{1,1}$ be the smallest ideal in $C^{\infty}(\mathcal{U})$ containing the functions $\left(\partial / \partial x^{\prime}\right)^{\gamma} S$ with $1 \leq|\gamma| \leq \mu$ and the functions $\left(\partial / \partial y^{\prime}\right)^{\delta} S$ with $1 \leq|\delta| \leq \nu$. For $(1,1) \prec(\mu, \nu)$ let $\mathcal{I}_{\mu, \nu}$ be the smallest ideal in $C^{\infty}(\mathcal{U})$ containing $\mathcal{I}_{1,1}$ and the functions $\bar{L} B(\bar{X}, \bar{Y})_{(x, y)}$ where $\bar{L}$ is a differential operator of type $\prec(\mu-1, \nu-1), \bar{X}$ is of type $(1,0)$ and $\bar{Y}$ is of type $(0,1)$. Then

(i) $\left[S_{u}^{\prime}\right]^{\mu} L_{x y} S-\mathcal{L}\left[B\left(X^{1}, Y^{1}\right)\right] \in \mathcal{I}_{\mu, \nu}$.

(ii) Let $\widetilde{X}=\sum_{j=1}^{n_{L}-1} \widetilde{a}_{j}(x, y) X_{j}$ and $\widetilde{Y}=\sum_{k=1}^{n_{R}-1} \widetilde{b}_{k}(x, y) Y_{k}$. If $g \in \mathcal{I}_{\mu, \nu}$, then $\widetilde{X} g \in \mathcal{I}_{\mu+1, \nu}$ and $\widetilde{Y} g \in \mathcal{I}_{\mu, \nu+1}$.

(iii) Suppose that $\mathcal{M}$ is not of type $\prec(\mu, \nu)$ at $P=(0,0)$. Then $L_{x y} S(0,0)=$ $\mathcal{L}\left[B\left(X^{1}, Y^{1}\right)\right](0,0)$ and this expression does not vanish if $\mathcal{M}$ is of type $(\mu, \nu)$.

Proof. We prove $(\mathrm{i})=(\mathrm{i})_{\mu, \nu}$ and (ii) $=(\text { ii })_{\mu, \nu}$ simultaneously by induction over $n=$ $\mu+\nu$. For $n=2$, i.e. $\mu=\nu=1$, the assertion (i) $)_{1,1}$ follows from (3.12).

Clearly $\widetilde{Y} S_{y_{k}} \in \mathcal{I}_{1,2}, \widetilde{X} S_{x_{j}} \in \mathcal{I}_{2,1}$. Next by (i) $)_{1,1}$ we have that $S_{u}^{\prime} S_{x_{j} y_{k}}^{\prime \prime}-$ $B\left(X_{j}, Y_{k}\right) \in \mathcal{I}_{1,1}$ and since $B\left(X_{j}, Y_{k}\right) \in \mathcal{I}_{1,2} \cap \mathcal{I}_{2,1}$ and $-S_{x_{j}} S_{u y_{k}}^{\prime \prime} \in \mathcal{I}_{1,1}$ we see that $X_{j} S_{y_{k}}^{\prime} \in \mathcal{I}_{2,1}$ and $Y_{k} S_{x_{j}}^{\prime} \in \mathcal{I}_{1,2}$. This implies also that $\widetilde{X} S_{y_{k}}^{\prime} \in \mathcal{I}_{2,1}$ and $\widetilde{Y} S_{x_{j}}^{\prime} \in \mathcal{I}_{1,2}$ and therefore (ii) for $\mu=\nu=1$. 
For the induction step let $\mu^{\prime}+\nu^{\prime}=n+1$. We consider the case $\mu^{\prime}>1$ and set $\mu=\mu^{\prime}-1, \nu^{\prime}=\nu$. Let $\mathcal{L}$ and $L_{x y}$ be as in (3.13), (3.14). Consider $\widetilde{\mathcal{L}}=\widetilde{X} \mathcal{L}$, and the constant coefficient operator $\widetilde{L}_{x y}=\left\langle\widetilde{\alpha}, \nabla_{x^{\prime}}\right\rangle\left\langle\alpha^{1}, \nabla_{x^{\prime}}\right\rangle \cdots\left\langle\beta^{m}, \nabla_{y^{\prime}}\right\rangle$ with $\widetilde{\alpha}=\widetilde{a}(x, y)$, $\alpha^{l}=a^{l}(x, y)$ and $\beta^{m}=b^{m}(x, y)$. Then $S_{u}^{\prime}\left\langle\widetilde{a}, \nabla_{x^{\prime}}\right\rangle-\sum_{j} \widetilde{a}_{j} X_{j}=\sum_{j} \widetilde{a}_{j} S_{x_{j}}^{\prime} \frac{\partial}{\partial u}$ and therefore

$$
\begin{aligned}
& \left(S_{u}^{\prime}\right)^{\mu+1}\left\langle\tilde{a}, \nabla_{x^{\prime}}\right\rangle L_{x y} S-\widetilde{X} \mathcal{L}\left[B\left(X^{1}, Y^{1}\right)\right] \\
& =\left(S_{u}^{\prime}\right)^{\mu} \sum_{j=1}^{n_{L}-1} \tilde{a}_{j} S_{x_{j}}^{\prime} \frac{\partial}{\partial u}\left(L_{x y} S\right)+\left(S_{u}^{\prime}\right)^{\mu} \widetilde{X}\left(\frac{1}{\left(S_{u}^{\prime}\right)^{\mu}}\right)\left(\left(S_{u}^{\prime}\right)^{\mu} L_{x y} S-\mathcal{L}\left(B\left(X^{1}, Y^{1}\right)\right)\right) \\
& +\widetilde{X}\left(\left(S_{u}^{\prime}\right)^{\mu} L_{x y} S-\mathcal{L}\left(B\left(X^{1}, Y^{1}\right)\right)\right)+\left(S_{u}^{\prime}\right)^{\mu} \widetilde{X}\left(\frac{1}{\left(S_{u}^{\prime}\right)^{\mu}}\right) \mathcal{L}\left(B\left(X^{1}, Y^{1}\right)\right) .
\end{aligned}
$$

The first term on the right hand side belongs to the ideal generated by the $S_{x_{j}}^{\prime}$, $1 \leq j \leq n_{L}-1$, hence to $\mathcal{I}_{1,1} \subset \mathcal{I}_{\mu+1, \nu}=\mathcal{I}_{\mu^{\prime}, \nu^{\prime}}$. The second belongs to $\mathcal{I}_{\mu, \nu}$ by the induction hypothesis for (i), hence also to $\mathcal{I}_{\mu^{\prime}, \nu^{\prime}}$. The fourth term involves $\mathcal{L}\left(B\left(X_{1}, Y_{1}\right)\right)$ and therefore also belongs to $\mathcal{I}_{\mu^{\prime}, \nu^{\prime}}$. The third term belongs to $\widetilde{X} \mathcal{I}_{\mu, \nu} \subset \mathcal{I}_{\mu+1, \nu}=\mathcal{I}_{\mu^{\prime}, \nu^{\prime}}$ by the induction hypothesis for (ii).

There is another case with $\mu^{\prime}+\nu^{\prime}=n+1$, namely $\nu^{\prime}>1$ and $\mu^{\prime}=\mu, \nu=$ $\nu^{\prime}-1$, and now $\widetilde{\mathcal{L}}$ is replaced by $\widetilde{Y} \mathcal{L}$ and the relevant constant coefficient operator is $\left\langle\widetilde{\beta}, \nabla_{y^{\prime}}\right\rangle\left\langle\alpha^{1}, \nabla_{x^{\prime}}\right\rangle \cdots\left\langle\beta^{m}, \nabla_{y^{\prime}}\right\rangle$ with $\widetilde{\beta}=\widetilde{b}(x, y), \alpha^{l}=a^{l}(x, y), \beta^{m}=b^{m}(x, y)$. One now shows that the difference of $\tilde{Y} \mathcal{L}\left(B\left(X^{1}, Y^{1}\right)\right)$ and the constant coefficient operator belongs to $\mathcal{I}_{\mu, \nu+1}=\mathcal{I}_{\mu^{\prime}, \nu^{\prime}}$. The argument is the same as before.

To complete the induction step we have to show (ii) $\mu_{\mu^{\prime}, \nu^{\prime}}$. We argue as in the first step. Assume that $\mu^{\prime}>1$. Clearly $\tilde{X}\left(\partial / \partial x^{\prime}\right)^{\gamma} S \in \mathcal{I}_{\mu^{\prime}, 1} \subset \mathcal{I}^{\mu^{\prime}, \nu^{\prime}}$ if $|\gamma|=\mu$. If $\delta$ is a multiindex with $|\delta|=\nu^{\prime}$, then by $(\mathrm{i})_{\left(1, \nu^{\prime}\right)}$ we see as in the first step that $\tilde{X}\left(\partial / \partial y^{\prime}\right)^{\delta} S \in \mathcal{I}_{2, \nu^{\prime}} \subset \mathcal{I}_{\mu^{\prime}, \nu^{\prime}}$. Also if $\bar{L}$ is a differential operator of type $\prec(\mu-1, \nu-1)$, then $\widetilde{X} \bar{L}$ is a differential operator of type $\prec(\mu, \nu-1)$. This shows $\widetilde{X} \mathcal{I}_{\mu^{\prime}-1, \nu^{\prime}} \subset \mathcal{I}_{\mu^{\prime}, \nu^{\prime}}$. Similarly one checks $\tilde{Y} \mathcal{I}_{\mu^{\prime}, \nu^{\prime}-1} \subset \mathcal{I}_{\mu^{\prime}, \nu^{\prime}}$, and the proof of the induction step is complete.

Finally, (iii) is an immediate consequence of (i) since if $\mathcal{M}$ is not of type $\prec(\mu, \nu)$ at $P$, then all functions in $\mathcal{I}_{\mu, \nu}$ vanish at the point $(0,0)$.

Given Lemma 3.5 we can refine Theorem 3.1 to

Theorem 3.6. Suppose $P=(x, y) \in \mathcal{M}$.

(1) The following conditions are equivalent.

(i) $\mathcal{M}$ is of type $(\mu, \nu)$ at $P$.

(ii) There are vector fields $X \in \mathcal{V}^{1,0}(P)$ and $Y \in \mathcal{V}^{0,1}(P)$ with the following property: If $L=V_{1} \cdots V_{\mu+\nu-2}$ where $\mu-1$ of the $V_{i}$ are equal to $X$ and $\nu-1$ of the $V_{i}$ are equal to $Y$, then $L[B(X, Y)]_{P} \neq 0$; but $\widetilde{L}[B(\widetilde{X}, \tilde{Y})]=0$ for all differential operators $\widetilde{L}$ of type $\prec(\mu-1, \nu-1)$ and all $\widetilde{X} \in \mathcal{V}^{1,0}(P), \widetilde{Y} \in \mathcal{V}^{0,1}(P)$.

(iii) There are vector fields $X \in \mathcal{V}^{1,0}(P)$ and $Y \in \mathcal{V}^{0,1}(P)$ with the following property: If $V=\operatorname{ad} V_{1} \cdots \operatorname{ad} V_{\mu+\nu-2}[X, Y]$ where $\mu-1$ of the $V_{i}$ are equal to $X$ and $\nu-1$ of the $V_{i}$ are equal to $Y$, then $\langle\omega, V\rangle_{P} \neq 0$; but $\langle\omega, \widetilde{V}\rangle_{P}=0$ for all vector fields $\widetilde{V}$ of type $\prec(\mu, \nu)$.

(2) Suppose $\mathcal{M}$ is not of type $\prec(\mu, \nu)$ at $P$. Then coordinates $x$ in $\mathfrak{X}, y$ in $\mathfrak{Y}$ vanishing at $P$ can be chosen near $P$, such that $\mathcal{M}$ is given by (3.6), so that (3.7) 
holds and

$$
\frac{\partial^{|\gamma|+|\delta|} S}{\left(\partial x^{\prime}\right)^{\gamma}\left(\partial x^{\prime}\right)^{\delta}}(0,0)=0
$$

for multiindices $\gamma, \delta$ with $(|\gamma|,|\delta|) \prec(\mu, \nu)$.

Moreover if $\mathcal{M}$ is of type $(\mu, \nu)$ at $P$, then the coordinates can be chosen so that in addition

$$
\frac{\partial^{\mu+\nu} S}{\left(\partial x_{1}\right)^{\mu}\left(\partial y_{1}\right)^{\nu}}(0,0) \neq 0 \text {. }
$$

Proof. The implications (ii) $\Longrightarrow$ (i), (iii) $\Longrightarrow$ (i) have already been proved in Theorem 3.1. Consider the vector space $\mathbb{V}$ of all linear combinations of monomials

$$
x_{1}^{\alpha_{1}} x_{2}^{\alpha_{2}} \cdots x_{n_{L}-1}^{\alpha_{n_{L}-1}} y_{1}^{\beta_{1}} y_{2}^{\beta_{2}} \cdots y_{n_{R}-1}^{\beta_{n_{R}-1}}
$$

with the property that $\sum_{j} \alpha_{j}=\mu, \sum_{k} \beta_{k}=\nu$. Then $\mathbb{V}$ is spanned by the polynomials of the form $\left\langle\xi^{\prime}, x^{\prime}\right\rangle^{\mu}\left\langle\eta^{\prime}, y^{\prime}\right\rangle^{\nu}, \xi^{\prime} \in \mathbb{R}^{n_{L}-1}, \eta^{\prime} \in \mathbb{R}^{n_{R}-1}$. This is seen by a straightforward adaptation of a corresponding argument in Stein's book [22, p.343] for polynomials of a single set of variables.

Now suppose that $\mathcal{M}$ is of type $(\mu, \nu)$ at $P$. Then we can choose coordinates such that $\mathcal{M}$ is given by (3.6), (3.7). The observation about $\mathbb{V}$ implies that

$$
\left\langle\xi^{\prime}, \nabla_{x^{\prime}}\right\rangle^{\mu-1}\left\langle\eta^{\prime}, \nabla_{y^{\prime}}\right\rangle^{\nu-1} S(0,0) \neq 0
$$

for suitable $\xi^{\prime} \in \mathbb{R}^{n_{L}-1}, \eta^{\prime} \in \mathbb{R}^{n_{R}-1}$. Then by Lemma 3.5 for $X_{\xi^{\prime}}:=\sum_{j=1}^{n_{L}-1} \xi_{j} X_{j}$, $Y_{\eta^{\prime}}:=\sum_{k=1}^{n_{R}-1} \eta_{k} Y_{k}$

$$
X_{\xi^{\prime}}^{\mu-1} Y_{\eta^{\prime}}^{\nu-1} B\left(X_{\xi^{\prime}}, Y_{\eta^{\prime}}\right) \neq 0
$$

moreover $\widetilde{L}[B(\widetilde{X}, \widetilde{Y})]=0$ for all $\widetilde{X} \in \mathcal{V}^{1,0}(P), \tilde{Y} \in \mathcal{V}^{0,1}(P)$ and all differential operators $\widetilde{L}$ of type $\prec(\mu-1, \nu-1)$, by Theorem 3.1. We have proved the implication (i) $\Longrightarrow$ (ii). The equivalence of the conditions (ii) and (iii) follows from Lemma 3.3. (3.15) follows from Lemma 3.5 and (3.16) can be achieved by separate rotations in the $x$ and the $y$ coordinates.

Corollary 3.7. Suppose $\operatorname{dim} \mathfrak{X}=\operatorname{dim} \mathfrak{Y}=2, \operatorname{dim} \mathcal{M}=3$ and $P \in \mathcal{M}$. Let $X \in \mathcal{V}^{1,0}(P)$ and $Y \in \mathcal{V}^{0,1}(P)$, nonvanishing at $P$. Then

(i) $\mathcal{M}$ is of type $(\mu, \nu)$ at $P$ if and only if

$$
\begin{aligned}
X^{\mu-1} Y^{\nu-1}\left(\operatorname{det} \mathcal{J}_{\Phi}\right)_{P} & \neq 0, \\
X^{\mu^{\prime}-1} Y^{\nu^{\prime}-1}\left(\operatorname{det} \mathcal{J}_{\Phi}\right)_{P} & =0 \text { for all }\left(\mu^{\prime}, \nu^{\prime}\right) \prec(\mu, \nu) .
\end{aligned}
$$

(ii) $\mathcal{M}$ is of type $(1, m-1)$ at $P=(x, y)$ if and only if $\left.\operatorname{det} J_{\Phi}\right|_{\mathcal{M}_{x}}$ vanishes of order $m-2$ at $y \in \mathcal{M}_{x}$.

(iii) $\mathcal{M}$ is of type $(m-1,1)$ at $P=(x, y)$ if and only if $\left.\operatorname{det} J_{\Phi}\right|_{\mathcal{M}^{y}}$ vanishes of order $m-2$ at $x \in \mathcal{M}^{y}$.

The proof is immediate from Theorem 3.1, Lemma 2.3 and Lemma 3.4. 


\section{4. $L^{p}$-ESTIMATES FOR INTEGRAL OPERATORS IN THE PLANE}

We assume that $\mathcal{M}$ is imbedded in $\mathbb{R}^{2} \times \mathbb{R}^{2}$ and that in a neighborhood of supp $\chi \cap \mathcal{M}$ the manifold $\mathcal{M}$ is given by $\Phi(x, y)=S\left(x_{1}, x_{2}, y_{1}\right)-y_{2}=0$ so that $S_{x_{2}}^{\prime} \neq 0$ and that

$$
y_{2}=S\left(x, y_{1}\right) \Longleftrightarrow x_{2}=\mathfrak{S}\left(y, x_{1}\right),
$$

with $S_{x_{2}}^{\prime} \neq 0$ and $\mathfrak{S}_{y_{2}}^{\prime} \neq 0$. Moreover we assume that $\mathcal{M}$ is of type $\preceq(\mu, \nu)$ at $P^{0} \in \operatorname{supp} \chi \cap \mathcal{M}$. Our operator is then defined by

$$
R f\left(x_{1}, x_{2}\right)=\int f\left(y_{1}, S\left(x, y_{1}\right)\right) \chi(x, y) d y_{1} .
$$

The Monge-Ampère determinant is now given by

$$
\operatorname{det} \mathcal{J}_{\Phi}(x, y)=S_{x_{1} y_{1}}^{\prime \prime}\left(x, y_{1}\right) S_{x_{2}}^{\prime}\left(x, y_{1}\right)-S_{x_{2} y_{1}}^{\prime \prime}\left(x, y_{1}\right) S_{x_{1}}^{\prime}\left(x, y_{1}\right)
$$

in particular it is independent of $y_{2}$.

Define vector fields $X, Y$ :

$$
\begin{aligned}
& X=\frac{\partial}{\partial x_{1}}+\mathfrak{S}_{x_{1}}^{\prime} \frac{\partial}{\partial x_{2}}, \\
& Y=\frac{\partial}{\partial y_{1}}+S_{y_{1}}^{\prime} \frac{\partial}{\partial y_{2}} .
\end{aligned}
$$

Then $X$ is of type $(1,0)$ and $Y$ is of type $(0,1)$ on $\mathcal{M}$. We note that the vector field

$$
T=\frac{\partial}{\partial y_{2}}+S_{x_{2}}^{\prime} \frac{\partial}{\partial x_{2}}
$$

is tangent to $\mathcal{M}$ and $X, Y$ and $T$ are linearly independent at any particular point $P \in \mathcal{M}$. An analogue to Lemma 2.2 is the formula

$$
[a X, b Y]=a X(b) Y-b Y(a) X+a b \Delta T+W
$$

where

$$
\Delta=\frac{\operatorname{det} \mathcal{J}_{\Phi}}{S_{x_{2}^{\prime}}}
$$

and $W=0$ on $\mathcal{M}$. It follows from Lemma 2.3 and Theorem 3.6 that the finite type condition can be restated in terms of $\Delta$. The assumption of type $\preceq(\mu, \nu)$ implies that there is $(j, k) \preceq(\mu-1, \nu-1)$ such that

$$
X^{j} Y^{k} \Delta \neq 0
$$

for $\left(x, y_{1}, S\left(x, y_{1}\right)\right)$ in supp $\chi \cap \mathcal{M}$.

Note that $\Delta$ does not depend on $y_{2}$. Therefore if

$$
\Gamma_{k}\left(y_{1}, y_{2}, x_{1}\right)=\frac{\partial^{k} \Delta}{\left(\partial y_{1}^{k}\right)}\left(x_{1}, \mathfrak{S}\left(y_{1}, y_{2}, x_{1}\right), y_{1}\right)
$$

then (4.7) just means that

$$
\frac{\partial^{j} \Gamma_{k}}{\left(\partial x_{1}\right)^{j}}\left(y_{1}, y_{2}, x_{1}\right) \neq 0
$$

for $\left(x_{1}, \mathfrak{S}\left(y, x_{1}\right), y_{1}\right)=\left(x, y_{1}, S\left(x, y_{1}\right)\right) \in \operatorname{supp} \chi \cap \mathcal{M}$.

We introduce a localization into regions where $\left|\operatorname{det} \mathcal{J}_{\Phi}\right| \approx|\Delta| \approx 2^{-l}$. Let

$$
\eta_{l}\left(x, y_{1}\right)=\eta\left(2^{l}\left|\Delta\left(x, y_{1}\right)\right|\right) \chi\left(x, y_{1}, S\left(x, y_{1}\right)\right)
$$


where $\eta \in C_{0}^{\infty}((1 / 2,2))$. We shall need $L^{p}$ estimates for the integral operator

$$
R_{l} f(x)=\int \eta_{l}\left(x, y_{1}\right) f\left(y_{1}, S\left(x, y_{1}\right)\right) d y_{1} .
$$

Proposition 4.1. Suppose that $\mathcal{M}$ is of type $\preceq(\mu, \nu)$ in $\operatorname{supp} \chi \cap \mathcal{M}$. Let $p=$ $\frac{\mu+\nu-2}{\mu-1}$ and suppose that $\gamma<(\mu+\nu-2)^{-1}$. Then the inequality

$$
\left\|R_{l} f\right\|_{p} \leq C_{\gamma} 2^{-l \gamma}\|f\|_{p}
$$

holds for all $f \in L^{p}$.

We shall use the following elementary

Lemma 4.2. Let $k$ and $N$ be positive integers, such that $k \leq N$. For an interval $J$ and $f \in C^{N}(J)$ let $M_{N}(f)=\max _{x \in J}\left|f^{(N)}(x)\right|$.

Then there is a constant $A_{k, N}$ such that for all $L$, for all intervals $J$ of length $L>0$, for all functions $f \in C^{N}(J)$ and for all $\beta, \gamma$ satisfying $\gamma \geq \frac{1}{N !}(L / 2)^{N} M_{N}(f)$, $\beta \geq \frac{2}{(N-k) !}(L / 2)^{N-k} M_{N}(f)$

$$
\operatorname{meas}\left\{x \in J:|f(x)| \leq \gamma,\left|f^{(k)}(x)\right| \geq \beta\right\} \leq A_{k, N}\left(\frac{\gamma}{\beta}\right)^{1 / k} .
$$

This estimate is an easy consequence of a lemma by M. Christ [1] which is closely related to van der Corput's Lemma on oscillatory integrals. It states that for any $k \in \mathbb{Z}_{+}$there is a constant $A_{k}$ such that for any interval $I \subset \mathbb{R}$, any $f \in C^{k}(I)$ and any $\gamma>0$

$$
\operatorname{meas}\{x \in I:|f(x)| \leq \gamma\} \leq A_{k} \gamma^{1 / k} \inf _{x \in I}\left|D^{k} f(x)\right|^{-1 / k} .
$$

Proof of Lemma 4.2. Let $a$ be the midpoint of $J$ and let $P$ be the Taylor polynomial of degree $N-1$ expanded about $x=a$. With the specifications on $\beta$ and $\gamma$ it follows that $|P(x)-f(x)| \leq \gamma,\left|P^{(k)}(x)-f^{(k)}(x)\right| \leq \beta / 2$ for all $x \in J$. If $E_{\beta, \gamma}$ denotes the set of all $x \in J$ with $|P(x)| \leq 2 \gamma$ and $\left|f^{(k)}(x)\right| \geq \beta / 2$, then

$$
\left\{x \in J:|f(x)| \leq \gamma,\left|f^{(k)}(x)\right| \geq \beta\right\} \subset E_{\beta, \gamma} .
$$

Since $P$ is a polynomial of degree $N-1$ the set $E_{\beta, \gamma}$ is the union of $O(N)$ disjoint intervals. We may apply Christ's estimate (4.9) to each of those intervals and as a result obtain

$$
\operatorname{meas}\left(E_{\beta, \gamma}\right) \leq C N A_{k}(4 \gamma / \beta)^{1 / k} .
$$

This implies the asserted inequality.

Proof of Proposition 4.1. Fix $N$ so that $2 N^{-1}<(\mu+\nu-2)^{-1}-\gamma$. Let $m_{N}$ be the maximum of the $C^{N+\nu}$ norm of $\Delta$ and the $C^{N+\mu}$ norm of $\Gamma_{k}$ in supp $\chi$. Recall that $\partial^{j} \Gamma_{k} /\left(\partial x_{1}\right)^{j} \neq 0$ for $\left(x, y_{1}, S\left(x, y_{1}\right)\right) \in \operatorname{supp} \chi$ where $(j, k) \preceq(\mu-1, \nu-1)$.

Let $A^{l}(x)=\left\{y_{1}:\left|\Delta\left(x, y_{1}\right)\right| \leq 2^{-l+1}\right\}$. If $\mu=1$, then $j=0$ so that $\partial_{y_{1}}^{k} \Delta \neq 0$ and hence by (4.9) $\left|A^{l}(x)\right| \leq C 2^{-l / k}$. The asserted $L^{\infty}$ estimate is immediate.

In what follows we assume that $\mu>1$ and $\nu>1$ (the case $\nu=1$ is easier). Let

$$
\begin{array}{r}
A_{m, l}(x)=\left\{y_{1}:\left|\Delta\left(x, y_{1}\right)\right| \leq 2^{-l+1}, 2^{-m} \leq\left|\frac{\partial^{k} \Delta}{\left(\partial y_{1}^{k}\right)}\left(x, y_{1}\right)\right| \leq 2^{-m+1},\right. \\
\left.\left(x, y_{1}, S\left(x, y_{1}\right)\right) \in \operatorname{supp} \chi\right\},
\end{array}
$$




$$
A_{l, l}(x)=\left\{y_{1}:\left|\frac{\partial^{k} \Delta}{\left(\partial y_{1}^{k}\right)}\left(x, y_{1}\right)\right| \leq 2^{-l+1},\left(x, y_{1}, S\left(x, y_{1}\right)\right) \in \operatorname{supp} \chi\right\}
$$

and

$$
B_{m}(y)=\left\{x_{1}:\left|\Gamma_{k}\left(y, x_{1}\right)\right| \leq 2^{-m+1},\left(x_{1}, \mathfrak{S}\left(y, x_{1}\right), y\right) \in \operatorname{supp} \chi\right\} .
$$

For any interval $J$ of length $2^{-l / N} m_{N}^{-1}$ the set $A_{m, l}(x) \cap J$ has measure $\leq$ $C 2^{(m-l) / k}$, by Lemma 4.2. Adding these estimates we obtain

$$
\operatorname{meas}\left(A_{m, l}(x)\right) \leq C_{N} 2^{l / N} 2^{(m-l) / k}
$$

uniformly in $x$. Similarly by (4.9)

$$
\operatorname{meas}\left(B_{m}(y)\right) \leq C_{j} 2^{-m / j}
$$

uniformly in $y$.

By Hölder's and Minkowski's inequalities

$$
\begin{aligned}
& \left(\int\left|R_{l} f(x)\right|^{p} d x\right)^{1 / p} \leq \sum_{m \leq l}\left(\int\left[\int_{A_{m, l}(x)}\left|f\left(y_{1}, S\left(x, y_{1}\right)\right)\right| d y_{1}\right]^{p} d x\right)^{1 / p} \\
& \quad \leq C_{N} 2^{l / N} \sum_{m \leq l} 2^{(m-l)(p-1) /(k p)}\left(\iint_{A_{m, l}(x)}\left|f\left(y_{1}, S\left(x, y_{1}\right)\right)\right|^{p} d y_{1} d x\right)^{1 / p} .
\end{aligned}
$$

Now note that if $y_{1} \in A_{m, l}(x)$ and $y_{2}=S\left(x, y_{1}\right)$ (or equivalently $x_{2}=\mathfrak{S}\left(y, x_{1}\right)$ ), then also $x_{1} \in B_{m}(y)$. We interchange the order of integration in (4.12) and perform the change of variable $y_{2}=\mathfrak{S}\left(x_{1}, x_{2}, y_{1}\right)$, for fixed $x_{1}, y_{1}$ :

$$
\begin{aligned}
& \iiint_{A_{m, l}(x)}\left|f\left(y_{1}, S\left(x, y_{1}\right)\right)\right|^{p} d y_{1} d x_{1} d x_{2} \\
& =\iiint_{\left\{x_{2}: y_{1} \in A_{m, l}\left(x_{1}, x_{2}\right)\right\}}\left|f\left(y_{1}, S\left(x, y_{1}\right)\right)\right|^{p} d x_{2} d x_{1} d y_{1} \\
& =\iiint_{\left\{y_{2}: y_{1} \in A_{m, l}\left(x_{1}, \mathfrak{S}\left(y_{1}, y_{2}, x_{1}\right)\right)\right\}}\left|f\left(y_{1}, y_{2}\right)\right|^{p}\left|\frac{\partial \mathfrak{S}}{\partial y_{2}}\right| d y_{2} d x_{1} d y_{1} \\
& \leq \iiint_{B_{m}(y)} d x_{1}\left|f\left(y_{1}, y_{2}\right)\right|^{p}\left|\frac{\partial \mathfrak{S}}{\partial y_{2}}\right| d y_{2} d y_{1} \\
& \leq C_{j} 2^{-m / j} \iint\left|f\left(y_{1}, y_{2}\right)\right|^{p} d y_{2} d y_{1}
\end{aligned}
$$

by (4.10). From (4.12) and (4.13) it follows that

$$
\begin{aligned}
\left\|R_{l} f\right\|_{p} & \leq C_{N} 2^{l / N} \sum_{-c \leq m \leq l} 2^{-\frac{m}{j p}} 2^{-\frac{l-m}{k}\left(1-\frac{1}{p}\right)}\|f\|_{p} \\
& \leq C_{N} 2^{l / N} \sum_{-c \leq m \leq l} 2^{-\frac{m}{(\mu-1) p}} 2^{-\frac{l-m}{\nu-1}\left(1-\frac{1}{p}\right)}\|f\|_{p} .
\end{aligned}
$$

If $p=p_{0}=(\mu+\nu-2) /(\mu-1)$, then this yields

$$
\left\|R_{l} f\right\|_{p_{0}} \leq C(1+l) 2^{l / N} 2^{-l /(\mu+\nu-2)}\|f\|_{p_{0}} \leq C_{\gamma} 2^{-l \gamma}\|f\|_{p_{0}} .
$$

Finally note that after notational changes the above argument also proves the easier $L^{1}$ estimate for the case $\nu=1$. 


\section{REgularity OF RAdON TRANSFORMS}

We shall first consider the case of Radon transforms associated with a family of curves in $\mathbb{R}^{2}$, where $\mathcal{M}$ is given by the equation $y_{2}=S\left(x, y_{1}\right)$; see (4.2). We assume that $S$ is defined in a neighborhood $\mathcal{U}$ of supp $\chi$.

Introducing a dyadic decomposition in the frequency variable we consider the operator

$$
R_{l}^{k} f(x)=\iint e^{i \tau\left(y_{2}-S\left(x, y_{1}\right)\right)} \eta\left(2^{l}\left|\Delta\left(x, y_{1}\right)\right|\right) a_{k}(x, y, \tau) f(y) d \tau d y
$$

where the $a_{k}$ are symbols of order 0 (with uniform bounds in $k$ ), so that $\tau \mapsto$ $a_{k}(x, y, \tau)$ is supported in the union of dyadic intervals $\pm\left[2^{k}, 2^{k+1}\right]$. The $C^{\infty}$ function $\eta$ is assumed to have support in $(1 / 2,2)$. The operator $R_{l}^{k}$ is bounded on $L^{p}\left(\mathbb{R}^{2}\right)$, uniformly in $l$ and $k$, since

$$
\left|R_{l}^{k} f(x)\right| \leq C_{N} \int 2^{k}\left(1+2^{k}\left|y_{2}-S\left(x, y_{1}\right)\right|\right)^{-N}\left|\eta_{l}\left(\Delta\left(x, y_{1}\right)\right)\right| f(y) d y .
$$

The main estimate of [19] is that

$$
\left\|R_{l}^{k} f\right\|_{2} \leq C_{\varepsilon} 2^{l(\varepsilon+1 / 2)} 2^{-k / 2}\|f\|_{2}
$$

This estimate is uniform in $S$, if $S$ varies over a compact subset of $C^{\infty}(\mathcal{U})$.

Let $R_{l}$ be defined as in (4.8).

Proposition 5.1. For $l \in \mathbb{Z}$

$$
\left\|R_{l} f\right\|_{L_{1 / 2}^{2}} \leq C_{\epsilon} 2^{l\left(\epsilon+\frac{1}{2}\right)}\|f\|_{2} .
$$

Proof. Let $\rho \in C_{0}^{\infty}\left(\mathbb{R}^{2}\right)$ be supported where $|\xi| \leq 1$ so that $\rho(\xi)=1$ if $|\xi| \leq 1 / 2$. Let $\beta_{0}=\rho$ and $\beta_{k}(\xi)=\rho\left(2^{-k-1}|\xi|\right)-\rho\left(2^{-k}|\xi|\right)$ for $k \geq 1$.

Suppose $l \leq k$. Then for a suitable choice of $a_{k}$ and a fixed integer $M$ (independent of $l$ and $k$ )

$$
\beta_{k}(D) R_{l}=\sum_{\kappa=k-M}^{k+M} \sum_{m=k-M}^{k+M} R_{l}^{\kappa} \beta_{m}(D)+E_{l}^{k}
$$

where

$$
\left\|E_{l}^{k}\right\|_{L^{2} \rightarrow L^{2}} \leq C_{N} 2^{-(k-l) N}
$$

(for the proof see estimate (5.7) in [19]). The inequalities (5.3) and (5.4) are used for $k \geq l(1+\varepsilon)$, and for $k \leq l(1+\varepsilon)$ we simply use the trivial bounds $\left\|\beta_{k}(D) R_{l}\right\|_{L^{2} \rightarrow L^{2}}=$ $O(1)$. We obtain

$$
\begin{aligned}
\left\|R_{l} f\right\|_{L_{1 / 2}^{2}} \leq C\left(\sum_{k} 2^{k}\left\|\beta_{k}(D) R_{l} f\right\|_{2}^{2}\right)^{1 / 2} & \\
\leq C_{1} 2^{l(1+\varepsilon) / 2}\|f\|_{2} & +C_{2} \sum_{n_{1}, n_{2}=-M}^{M}\left(\sum_{k \geq l(1+\varepsilon)} 2^{k}\left\|R_{l}^{k+n_{1}} \beta_{k+n_{2}}(D) f\right\|_{2}^{2}\right)^{1 / 2} \\
& +C_{3}\left(\sum_{k \geq l(1+\varepsilon)} 2^{k}\left\|E_{l}^{k} f\right\|_{2}^{2}\right)^{1 / 2} .
\end{aligned}
$$


By (5.3) and an orthogonality argument the second expression is dominated by

$$
C 2^{l(\varepsilon+1 / 2)} \sum_{n_{2}=-M}^{M}\left(\sum_{k}\left\|\beta_{k+n_{2}}(D) f\right\|_{2}^{2}\right)^{1 / 2} \leq C^{\prime} 2^{l(\varepsilon+1 / 2)}\|f\|_{2}
$$

while the third expression is bounded by

$$
C \sum_{k \geq l(1+\varepsilon)} 2^{(k-2 k N) / 2} 2^{l N}\|f\|_{2} \leq C^{\prime} 2^{l / 2}\|f\|_{2} .
$$

The assertion follows.

Proof of Theorem 1.3. By Lemma 2.3 and (2.6) we have

$$
\langle\omega,[X, Y]\rangle_{(x, y)}=a(x, y) \Delta(x, y)
$$

where $a$ is a $C^{\infty}$ function, nonvanishing in $\mathcal{U}$. Let $\chi_{\gamma}=|a|^{\gamma} \chi$; then $\tilde{\chi}_{\gamma}$ is smooth and the asserted estimate follows if we can show that for $\gamma>1 / 2$ the operator

$$
R_{\gamma} f(x)=\int\left|\Delta\left(x, y_{1}\right)\right|^{\gamma} \chi_{\gamma}\left(x, y_{1}, S\left(x, y_{1}\right)\right) f\left(y_{1}, S\left(x, y_{1}\right)\right) d y_{1}
$$

maps $L^{2}$ boundedly to $L_{1 / 2}^{2}$.

Let $\eta \in C_{0}^{\infty}(1 / 2,2)$ so that $\sum_{l=-\infty}^{\infty} \eta\left(2^{l} s\right)=1$ for $s>0$. Define $\widetilde{\eta}_{\gamma}(s)=\eta(s)|s|^{\gamma}$ (so that $\eta_{\gamma}$ is an admissible cutoff function in (5.1)). Define

$$
R_{\gamma, l} f(x)=\int \widetilde{\eta}_{\gamma}\left(2^{l}\left|\Delta\left(x, y_{1}\right)\right|\right) \chi_{\gamma}\left(x, y_{1}, S\left(x, y_{1}\right)\right) f\left(y_{1}, S\left(x, y_{1}\right)\right) d y_{1} .
$$

The operator $R_{\gamma, l}$ maps $L^{2}$ to $L_{1 / 2}^{2}$ with norm bounded by $C_{\varepsilon} 2^{l(\varepsilon+1 / 2)}$, by Proposition 5.1. However $R_{\gamma}=\sum_{l} 2^{-l \gamma} R_{\gamma, l}$ and $R_{\gamma, l}=0$ for large negative $l$, so the asserted bound for $R_{\gamma}$ follows.

Estimates for Radon transforms associated with hypersurfaces. Assume now that $\mathcal{M}$ is a hypersurface in $\mathfrak{X} \times \mathfrak{Y}$. Suppose that $(\mu, \nu) \in \tau(P)$ (that is, $\mathcal{M}$ is of type $(\mu, \nu)$ at $P)$. Then there is a neighborhood $\mathcal{U}$ so that $\mathcal{M}$ is of type $\preceq(\mu, \nu)$ in $\mathcal{U}$ and we assume that the cutoff function $\chi$ is supported in $\mathcal{U}$.

The $L^{p}$ Sobolev estimates of Theorem 1.1 follow from the following result by multiple interpolation.

Proposition 5.2. Let $p=(\mu+\nu) / \mu, \alpha<(\mu+\nu)^{-1}$. Then $R$ maps $L_{\text {comp }}^{p}(\mathfrak{Y})$ boundedly to $L_{\alpha, \text { loc }}^{p}(\mathfrak{X})$.

Proof. A duality argument shows that it is sufficient to consider the case $\mu \leq \nu$, and since the nondegenerate case is well understood we may assume that $\nu \geq 2$. We may choose coordinates $x$ in $\mathfrak{X}$ and $y$ in $\mathfrak{Y}$ so that $x(P)=0, y(P)=0$ and $\mathcal{M}$ is given by (3.6), (3.7) near $P$. Let

$$
R^{k} f\left(x^{\prime}, v\right)=\iiint e^{i \tau\left(v-S\left(x^{\prime}, u, y^{\prime}\right)\right)} a_{k}(x, y, \tau) d \tau f\left(y^{\prime}, v\right) d y^{\prime} d v
$$

where $a_{k}(x, y, \tau)=\beta_{k}(\tau) \chi(x, y)$ is a symbol of order 0 supported where $|\tau| \approx 2^{k}$. Since we do not attempt to prove endpoint results it suffices to show that the $L^{p} \rightarrow L^{p}$ bound of $R^{k}$ is $O\left(2^{-k \alpha}\right)$ for $\alpha<(\mu+\nu)^{-1}$ and $p=(\mu+\nu) / \mu$. Let $X_{1}, Y_{1}$ be as in (3.8), (3.9), and by Theorem 3.6 we may assume that

$$
X_{1}^{\mu-1} Y_{1}^{\nu-1} B\left(X_{1}, Y_{1}\right) \neq 0
$$


where

$$
B\left(X_{1}, Y_{1}\right)=S_{u}^{\prime} S_{x_{1} y_{1}}^{\prime \prime}-S_{x_{1}}^{\prime} S_{u y_{1}}^{\prime \prime}
$$

Split $x^{\prime}=\left(x_{1}, x^{\prime \prime}\right), y^{\prime}=\left(y_{1}, y^{\prime \prime}\right)$ and define for functions $g\left(y_{1}, v\right)$

$$
\begin{aligned}
& R_{x^{\prime \prime} y^{\prime \prime}}^{k} g\left(x_{1}, u\right)= \\
& \quad \iiint e^{i \tau\left(v-S\left(x_{1}, x^{\prime \prime}, u, y_{1}, y^{\prime \prime}\right)\right)} a_{k}\left(x_{1}, x^{\prime \prime}, u, y_{1}, y^{\prime \prime}, v, \tau\right) d \tau g\left(y_{1}, v\right) d y_{1} d v .
\end{aligned}
$$

Moreover put $\Delta=B\left(X_{1}, Y_{1}\right) / S_{u}^{\prime}$, then also $X_{1}^{\mu-1} Y_{1}^{\nu-1} \Delta \neq 0$. Define

$$
\zeta_{l}\left(x_{1}, x^{\prime \prime}, u, y_{1}, y^{\prime \prime}, v\right)=\eta\left(2^{l}\left|\Delta\left(x_{1}, x^{\prime \prime}, u, y_{1}, y^{\prime \prime}\right)\right|\right) \chi\left(x_{1}, x^{\prime \prime}, u, y_{1}, y^{\prime \prime}, v\right),
$$

where $\eta$ satisfies $\sum_{l} \eta\left(2^{l} s\right)=1$ for $s>0$, and

$$
\begin{aligned}
& R_{x^{\prime \prime} y^{\prime \prime}, l}^{k} g\left(x_{1}, u\right)=\iiint e^{i \tau\left(v-S\left(x_{1}, x^{\prime \prime}, u, y_{1}, y^{\prime \prime}\right)\right)} \zeta_{l} \beta_{k}(\tau) d \tau g\left(y_{1}, v\right) d y_{1} d v \\
& R_{x^{\prime \prime} y^{\prime \prime}, l} g\left(x_{1}, u\right)=\int \zeta_{l} \chi\left(x_{1}, x^{\prime \prime}, u, y_{1}, y^{\prime \prime}, S\left(x_{1}, x^{\prime \prime}, u, y_{1}, y^{\prime \prime}\right)\right) g\left(y_{1}, v\right) d y_{1}
\end{aligned}
$$

By $(5.3)$

$$
\left\|R_{x^{\prime \prime} y^{\prime \prime}, l}^{k} g\right\|_{L^{2}\left(\mathbb{R}^{2}\right)} \leq C 2^{-k / 2} 2^{l(\varepsilon+1 / 2)}\|g\|_{L^{2}\left(\mathbb{R}^{2}\right)}
$$

uniformly in $x^{\prime \prime}, y^{\prime \prime}$. By $(5.1)$ we have

$$
\left|R_{x^{\prime \prime} y^{\prime \prime}}^{k} g\right| \leq C_{N} \int \frac{2^{k}}{1+2^{k}|s|}\left|R_{x^{\prime \prime} y^{\prime \prime}, l}[g(\cdot, \cdot+s)]\right| d s
$$

and from Proposition 4.1 it follows that for $p_{0}=(\mu+\nu-2) /(\mu-1)>2$

$$
\left\|R_{x^{\prime \prime} y^{\prime \prime}, l}^{k} g\right\|_{L^{p_{0}\left(\mathbb{R}^{2}\right)}} \leq C_{\gamma} 2^{-l \gamma}\|g\|_{L^{p_{0}\left(\mathbb{R}^{2}\right)}}, \quad \gamma<(\mu+\nu-2)^{-1} .
$$

Since $R_{x^{\prime \prime} y^{\prime \prime}, l}^{k}$ is $L^{1} \rightarrow L^{1}$ bounded with uniform bound in $l, k, x^{\prime \prime}, y^{\prime \prime}$ we also obtain that

$$
\left\|R_{x^{\prime \prime} y^{\prime \prime}, l}^{k} g\right\|_{L^{r}\left(\mathbb{R}^{2}\right)} \leq C_{\beta} 2^{-l \beta}\|g\|_{L^{r}\left(\mathbb{R}^{2}\right)}, \quad \beta<\frac{1-r^{-1}}{1-p_{0}^{-1}} \frac{1}{\mu+\nu-2}, \quad 1<r<p_{0} .
$$

Interpolation of (5.7) and (5.8) yields that for $p=(\mu+\nu) / \mu \in\left(2, p_{0}\right)$

$$
\left\|R_{x^{\prime \prime} y^{\prime \prime}, l}^{k} g\right\|_{L^{p}\left(\mathbb{R}^{2}\right)} \leq C_{\epsilon} 2^{-k /(\mu+\nu)} 2^{l \varepsilon}\|g\|_{L^{p}\left(\mathbb{R}^{2}\right)} .
$$

We take a suitable geometric mean of (5.9) and (5.10) and obtain that

$$
\left\|R_{x^{\prime \prime} y^{\prime \prime}, l}^{k} g\right\|_{L^{p}\left(\mathbb{R}^{2}\right)} \leq C_{\alpha} 2^{-l \epsilon(\alpha)} 2^{-k \alpha}\|g\|_{L^{p}\left(\mathbb{R}^{2}\right)}
$$

where $\alpha<(\mu+\nu)^{-1}$ and $\varepsilon(\alpha)>0$ in this range of $\alpha$. Observe that

$$
R^{k} f(x)=\sum_{l} \int R_{x^{\prime \prime} y^{\prime \prime}, l}^{k}\left[f\left(\cdot, y^{\prime \prime}, \cdot\right)\right] d y^{\prime \prime}
$$

Since supp $\chi$ is compact we see from (5.11) and applications of Minkowski's and Hölder's inequalities that

$$
\left\|R^{k}\right\|_{L^{p} \rightarrow L^{p}}=O\left(2^{-k \alpha}\right)
$$

for $\alpha<(\mu+\nu)^{-1}$.

We now use the Sobolev estimates to deduce $L^{p} \rightarrow L^{q}$ estimates. The argument follows known patterns. 
Lemma 5.3. Suppose that $R$ is bounded from $L^{p_{i}}$ to $L_{\alpha_{i}}^{p_{i}}$, with $\alpha_{i}>0$, $i=$ $1, \ldots, N$. Suppose $(1 / p, 1 / q)$ belongs to the interior of the convex hull of the points $\left(\left(\alpha_{i}+p_{i}^{-1}\right) /\left(1+\alpha_{i}\right), p_{i}^{-1} /\left(1+\alpha_{i}\right)\right)$. Then $R$ is bounded from $L_{\text {comp }}^{p}$ to $L_{\mathrm{loc}}^{q}$.

Proof. One uses the obvious fact that for $k>0$ the $L^{1} \rightarrow L^{\infty}$ bound of $R^{k}$ is $O\left(2^{k}\right)$ (this does not rely on any finite type assumption). The assumed boundedness in Sobolev spaces implies that the $L^{p_{i}} \rightarrow L^{p_{i}}$ operator norm is $O\left(2^{-k \alpha_{i}}\right)$. An interpolation shows that the $L^{p} \rightarrow L^{q}$ operator norm of $R^{k}$ is bounded by $C 2^{-k \varepsilon(p, q)}$ with $\varepsilon(p, q)>0$ if $(1 / p, 1 / q)$ belongs to the half open line segment connecting the points $\left(1 / p_{i}, 1 / p_{i}\right)$ and $\left(\left(\alpha_{i}+p_{i}^{-1}\right) /\left(1+\alpha_{i}\right), p_{i}^{-1} /\left(1+\alpha_{i}\right)\right)$ excluding the latter. Interpolating with the trivial $L^{1} \rightarrow L^{1}$ and $L^{\infty} \rightarrow L^{\infty}$ estimates yields $L^{p} \rightarrow L^{q}$ boundedness for $(1 / p, 1 / q)$ in the interior of the triangle with corners $(0,0),(1,1)$, $\left(\left(\alpha_{i}+p_{i}^{-1}\right) /\left(1+\alpha_{i}\right), p_{i}^{-1} /\left(1+\alpha_{i}\right)\right)$ and obvious further interpolations yield the assertion.

The Lemma applied with $p_{i}=\left(\mu_{i}+\nu_{i}\right) / \mu_{i}, \alpha_{i}=\left(\mu_{i}+\nu_{i}\right)^{-1}$ and then $\left(\alpha_{i}+p_{i}^{-1}\right) /\left(1+\alpha_{i}\right)=(\mu+1) /(\mu+\nu+1), p_{i}^{-1} /\left(1+\alpha_{i}\right)=\mu /(\mu+\nu+1)$ implies the $L^{p} \rightarrow L^{q}$ inequalities asserted in Theorem 1.2.

We conclude with an observation concerning the boundedness regions $\mathcal{A}(P)$ and $\mathcal{B}(P)$, mentioned in the introduction.

Lemma 5.4. The regions $\overline{\mathcal{A}}(P), \underline{\mathcal{A}}(P)$ and $\overline{\mathcal{B}}(P), \underline{\mathcal{B}}(P)$ are convex polygonal regions. They have at least three extreme points if and only if $\mathcal{M}$ is of finite type at $P$. In this case let $n=\min \{\mu+\nu:(\mu, \nu) \in \tau(P)\}$ be the Hörmander type of $\mathcal{M}$ at $P$. Then there are at most $n-1$ different pairs $\left(\mu_{s}, \nu_{s}\right)$ so that $\mathcal{M}$ is of type $\left(\mu_{s}, \nu_{s}\right)$ at $\mathcal{M}$, and the regions $\overline{\mathcal{A}}(P), \underline{\mathcal{A}}(P), \overline{\mathcal{B}}(P), \underline{\mathcal{B}}(P)$ have at most $n+1$ extreme points.

Proof. Fix a pair $(\mu, \nu) \in \tau(P)$ so that $\mu+\nu=n$. Let $E_{1}$ consist of those integers $j$ with $1 \leq j \leq \mu$ such that $(j, k) \in \tau(P)$ for some $k \geq 1$. Let $E_{2}$ consist of those integers $k$ with $1 \leq k \leq \nu-1$ such that $(j, k) \in \tau(P)$ for some $j \geq 1$. For $j \in E_{1}$ let $k_{j}=\min \{k:(j, k) \in \tau(P)\}$ and for $k \in E_{2}$ let $j_{k}=\min \{j:(j, k) \in \tau(P)\}$. If $\mathcal{M}$ is of type $\left(\mu_{s}, \nu_{s}\right)$ at $P$, then $\left(\mu_{s}, \nu_{s}\right)$ has to be one of the points $\left(j, k_{j}\right)$, $j \in E_{1}$, or $\left(j_{k}, k\right), k \in E_{2}$, and there are at most $n-1$ such points. Moreover it is easy to see that $\overline{\mathcal{A}}(P)$ is the convex hull of the points $(u, \beta)$ with $\beta \leq 0$ and $0 \leq u \leq 1$ and the points $(0,0),(1,0),\left(j /\left(j+k_{j}\right), 1 /\left(j+k_{j}\right)\right), j \in E_{1}$, and $\left(j_{k} /\left(j_{k}+k\right), 1 /\left(j_{k}+k\right)\right), k \in E_{2}$. Every extreme point of $\overline{\mathcal{A}}(P)$ has to be among these points. Similarly $\underline{\mathcal{B}}(P)$ is the convex hull of the points $(u, v)$ with $v \geq u$ and $0 \leq u \leq 1$ and the points $(0,0),(1,0),\left((j+1) /\left(j+k_{j}+1\right), j /\left(j+k_{j}+1\right)\right), j \in E_{1}$, and $\left(\left(j_{k}+1\right) /\left(j_{k}+k+1\right), j_{k} /\left(j_{k}+k+1\right)\right), k \in E_{2}$, and every extreme point of $\overline{\mathcal{B}}(P)$ has to be among these points. A similar consideration applies to $\underline{\mathcal{A}}(P), \underline{\mathcal{B}}(P)$.

\section{NeCESSARY CONDITIONS}

Fixing $P$, we may introduce coordinates vanishing at $P$, so that (3.6) and (3.7) hold. From Taylor's formula we obtain

$$
S\left(x^{\prime}, x_{n_{L}}, y^{\prime}\right)=\sum_{|\alpha| \leq N} \sum_{|\beta| \leq N} \rho_{\alpha \beta}\left(x^{\prime}\right)^{\alpha}\left(y^{\prime}\right)^{\beta}+O\left(\left(\left|y^{\prime}\right|+\left|x^{\prime}\right|\right)^{N+1}+\left|x_{n_{L}}\right|\right),
$$

and if $\mathcal{M}$ is of not of type $(j, k)$ at $P$, for $(j, k) \prec(\mu, \nu)$, then

$$
\rho_{\alpha \beta}=0 \text { if }(|\alpha|,|\beta|) \prec(\mu, \nu)
$$

by Theorem 3.6. 
In what follows we use the notation

$$
c_{j k}=\left(\frac{\frac{j}{n_{L}-1}+1}{\frac{j}{n_{L}-1}+\frac{k}{n_{R}-1}+1}, \frac{\frac{j}{n_{L}-1}}{\frac{j}{n_{L}-1}+\frac{k}{n_{R}-1}+1}\right) .
$$

Note that except for $(0,0)$ and $(1,1)$ all the extreme points of the region $\underline{\mathcal{A}}(P)$ are of the form $c_{\mu \nu}$ for suitable integers $\mu \geq 1, \nu \geq 1$.

It suffices to show the necessity of the condition $(1 / p, 1 / q) \in \underline{\mathcal{B}}(P)$ in Theorem 1.2 since it implies the necessity of the condition $(1 / p, \alpha) \in \underline{\mathcal{A}}(P)$ in Theorem 1.1, by Lemma 5.3 and a simple computation.

Lemma 6.1. Assume $P \in \mathcal{M}$ and $\chi(P) \neq 0$. Suppose that $1 \leq p \leq \infty$ and that $R$ is bounded from $L^{p} \rightarrow L^{q}$. Then

(i) $n_{R} / p \leq 1 / q+n_{R}-1$ so that $(1 / p, 1 / q)$ is on or above the line connecting $c_{\mu 1}$ and $(1,1)$, for all $\mu>0$, and

(ii) $q \leq n_{L} p$, so that $(1 / p, 1 / q)$ is on or above the line connecting $c_{1 \nu}$ and $(0,0)$.

(iii) Suppose that $\mathcal{M}$ is not of type $\left(\mu^{\prime}, \nu^{\prime}\right)$ for all $\left(\mu^{\prime}, \nu^{\prime}\right)$ with either $\mu^{\prime}<\mu$ and $\nu^{\prime}>0$ or $\mu^{\prime}=\mu$ and $\nu^{\prime}<\nu$. Then $\frac{n_{R}-1}{\nu}+\frac{1}{q} \geq \frac{n_{R}-1+\nu}{\nu p}$, i.e. $(1 / p, 1 / q)$ is on or above the line connecting $c_{\mu \nu}$ and $(1,1)$.

(iv) Suppose that $\mathcal{M}$ is not of type $\left(\mu^{\prime}, \nu^{\prime}\right)$ for all $\left(\mu^{\prime}, \nu^{\prime}\right)$ with either $\mu^{\prime}>0$ and $\nu^{\prime}<\nu$ or $\mu^{\prime}<\mu$ and $\nu^{\prime}=\nu$. Then $q \leq\left(n_{L}-1+\mu\right) p / \mu$, i.e. $(1 / p, 1 / q)$ is on or above the line connecting $c_{\mu \nu}$ and $(0,0)$.

(v) Suppose that $\mathcal{M}$ is not of finite type at $P$. Then $p \geq q$.

(vi) Suppose that $c_{\mu \nu}$ and $c_{\mu^{\prime} \nu^{\prime}}$ are two extreme points of $\mathcal{B}(P)$ (different from $(0,0),(1,1))$, so that the line segment connecting $c_{\mu \nu}$ and $c_{\mu^{\prime} \nu^{\prime}}$ is an edge of $\mathcal{B}(P)$. Then $(1 / p, 1 / q)$ lies on or above the straight line through $c_{\mu \nu}$ and $c_{\mu^{\prime} \nu^{\prime}}$.

Proof. For assertion (i) one chooses $f=\chi_{\delta}$ to be the characteristic function of a ball of radius $\delta$. Then $|R f(x)| \geq c \delta^{n_{R}-1}$ on a set of measure $\geq c_{1} \delta$ and therefore $\left\|R f_{\delta}\right\|_{q} \geq \delta^{n_{R}-1+1 / q}$ while $\left\|f_{\delta}\right\|_{p}=O\left(\delta^{n_{R} / p}\right)$. (ii) follows from (i) by applying the first to the adjoint operator. Similarly (iv) follows from (iii).

To see (iii) we assume that $P=(0,0)$ and assume that $\mathcal{M}$ near $P$ is given by (3.6), (3.7) with $u=x_{2}, v=y_{2}$. We use (6.1) for large $N$. Let $f$ be the characteristic function of the cylinder $\left\{\left|y^{\prime}\right| \leq c_{1} \delta^{1 / \nu},\left|y_{n_{R}}\right| \leq \delta\right\}$. Then $|R f(x)| \geq c \delta^{\left(n_{R}-1\right) / \nu}$ if $\left|x^{\prime}\right| \leq c_{2} \delta^{1 /(N+1)}$ (here we apply (6.1), (6.2)) and $\left|x_{n_{L}}\right| \leq c_{3} \delta$ for suitable $c_{1}$, $c_{2}, c_{3}$. Therefore $\|R f\|_{q} \geq \delta^{\left(n_{R}-1\right) / \nu} \delta^{\left(N+n_{R}\right) /(N+1) q}$, but $\left\|f_{\delta}\right\|_{p} \leq C \delta^{\left(n_{R}-1+\nu\right) / \nu p}$. Therefore the assumed $L^{p} \rightarrow L^{q}$ boundedness yields the restriction $\left(n_{R}-1\right) / \nu+$ $\left(N+n_{R}\right) / q(N+1) \geq\left(n_{R}-1+\nu\right) /(\nu p)$. (iii) follows from letting $N \rightarrow \infty$. Moreover (v) follows immediately from a combination of (i)-(iv).

Finally we turn to the proof of (vi). Let $\widetilde{\mu}=\frac{\mu}{n_{L}-1}, \widetilde{\nu}=\frac{\nu}{n_{R}-1}$. Suppose that $c_{\mu \nu}$ and $c_{\mu^{\prime} \nu^{\prime}}$ are two extreme points of $\underline{\mathcal{B}}(P)$ so that $c_{\mu \nu}$ lies to the left of $c_{\mu^{\prime} \nu^{\prime}}$, that is, $(\widetilde{\mu}+1) /(\widetilde{\mu}+\widetilde{\nu}+1) \leq\left(\widetilde{\mu}^{\prime}+1\right) /\left(\widetilde{\mu}^{\prime}+\widetilde{\nu}^{\prime}+1\right)$; this condition is equivalent with $(\widetilde{\mu}+1) \widetilde{\nu}^{\prime}<\left(\widetilde{\mu}^{\prime}+1\right) \widetilde{\nu}$. Since $c_{\mu \nu}$ is an extreme point it lies below the line through $(0,0)$ and $c_{\mu^{\prime} \nu^{\prime}}$ which implies that $\widetilde{\mu}<\widetilde{\mu}^{\prime}$. Since $c_{\mu^{\prime} \nu^{\prime}}$ is an extreme point it lies below the line through $c_{\mu \nu}$ and $(1,1)$ which implies that $\widetilde{\nu}^{\prime}<\widetilde{\nu}$. Both conditions also imply that $\widetilde{\mu}^{\prime} \widetilde{\nu}-\widetilde{\mu} \widetilde{\nu}^{\prime}>0$. Let

$$
a=\frac{1}{n_{L}-1} \frac{\widetilde{\nu}-\widetilde{\nu}^{\prime}}{\widetilde{\mu}^{\prime} \widetilde{\nu}-\widetilde{\mu}^{\prime}}, \quad b=\frac{1}{n_{R}-1} \frac{\widetilde{\mu}^{\prime}-\widetilde{\mu}}{\widetilde{\mu}^{\prime} \widetilde{\nu}-\widetilde{\mu} \widetilde{\nu}^{\prime}} .
$$

A computation shows that the point $(u, v)$ is below the line through $c_{\mu \nu}$ and $c_{\mu^{\prime} \nu^{\prime}}$ if and only if $\left(b\left(n_{R}-1\right)+1\right) u-\left(a\left(n_{L}-1\right)+1\right) v>b\left(n_{R}-1\right)$. Moreover the point 
$c_{j k}$ is below this line if and only if $a j+b k<1$. Therefore, $\mathcal{M}$ is not of finite type $(j, k)$ if $a j+b k<1$, by the definition of $\underline{\mathcal{B}}(P)$.

Now let $f_{\delta}$ be the characteristic function of the cylinder $\left\{\left|y^{\prime}\right| \leq \delta^{b},\left|y_{n_{R}}\right| \leq \delta\right\}$. By (6.1) with $N \gg a, b$ we see that

$$
\left|S\left(x, y^{\prime}\right)\right| \leq C \sum_{\substack{(j, k) \in \tau(P) \\ 1 \leq j \leq N \\ 1 \leq k \leq N}}\left|x^{\prime}\right|^{j} \delta^{k b}+O\left(\delta^{b(N+1)}+\left|x^{\prime}\right|^{N+1} \delta^{b}+\left|x_{n_{L}}\right|\right) .
$$

We choose $N$ large and evaluate for $\left|x^{\prime}\right| \leq c_{1} \delta^{a},\left|x_{n_{L}}\right| \leq c_{2} \delta$ to see that $\left|R f_{\delta}(x)\right| \geq$ $\delta^{b\left(n_{R}-1\right)}$ on a set of measure $>c_{2} \delta^{a\left(n_{L}-1\right)+1}$. Therefore

$$
\left\|R f_{\delta}\right\|_{q} \geq c \delta^{b\left(n_{R}-1\right)+\left(a\left(n_{L}-1\right)+1\right) / q}
$$

while $\left\|f_{\delta}\right\|_{p}=O\left(\delta^{\left(b\left(n_{R}-1\right)+1\right) / p}\right)$. Assuming that $R$ is bounded from $L^{p}$ to $L^{q}$ and letting $\delta \rightarrow 0$ we obtain the restriction $\left(b\left(n_{R}-1\right)+1\right) / p-\left(a\left(n_{L}-1\right)+1\right) / q \leq b\left(n_{R}-1\right)$ and this is precisely the assertion.

\section{Appendix}

We have noticed that our argument used in [19, Lemma 3.6] is correct only in the semitranslation-invariant case. Since this lemma is a technical step leading to inequality (5.3) above we give the proof valid in the general case in this appendix.

Let $\rho$ denote a $C^{\infty}$ function of the variables $\left(w, \tau, y_{1}\right) \in \mathbb{R}^{2} \times \mathbb{R} \times \mathbb{R}$, supported near $\left(w, y_{1}, \tau\right)_{0}=(0,0,1)$, in particular $\rho\left(w, \tau, y_{1}\right)=0$ for $\tau<1 / 2$. Let $\left(w, y_{1}\right) \mapsto$ $S\left(w, y_{1}\right)$ be a $C^{\infty}$ function such that $1 / 4 \leq \partial_{w_{2}} S \leq 4$. We assume that if $w, z \in$ supp $\rho\left(\cdot, \tau, y_{1}\right)$ for any $\tau, y_{1}$, then the equation $S\left(z, y_{1}\right)-S\left(w, y_{1}\right)=0$ can be solved in $z_{1}$ and in $w_{1}$; that is, there is a function $v$ such that

$$
S\left(z_{1}, v\left(z_{1}, w, y_{1}\right), y_{1}\right)-S\left(w, y_{1}\right)=S\left(z, y_{1}\right)-S\left(w_{1}, v\left(w_{1}, z, y_{1}\right)\right)=0 .
$$

Note that then

$$
S\left(z, y_{1}\right)-S\left(w, y_{1}\right) \approx z_{2}-v\left(z_{1}, w, y_{1}\right) \approx v\left(w_{1}, z, y_{1}\right)-w_{2} .
$$

Let $n$ be a fixed positive integer and $r=\left(r_{1}, \ldots, r_{n}\right) \in \mathbb{Z}^{n}$ such that $0 \leq r_{i} \leq$ $l+n+C$ for suitable $C$. Let

$$
\sigma_{j}\left(x, y_{1}\right)=\left.\frac{1}{(j+1) !}\left(\frac{\partial}{\partial z_{1}}\right)^{j+1}\left[S_{y_{1}}^{\prime}\left(z_{1}, v\left(z_{1}, x, y_{1}\right), y_{1}\right)\right]\right|_{z_{1}=y_{1}} .
$$

Let $\chi_{l}^{0}(s)=\chi_{0}\left(2^{l+n+4} s\right)$ and $\chi_{l}^{r_{i}}(s)=\chi_{0}\left(2^{l-r_{i}+n+4} s\right)-\chi_{0}\left(2^{l-r_{i}+n+3} s\right)$ and let $\gamma_{l}^{r}\left(x, y_{1}\right)=\chi_{l}\left(\sigma_{0}\left(x, y_{1}\right)\right) \prod_{i=1}^{n} \chi_{l}^{r_{i}}\left(\sigma_{i}\left(x, y_{1}\right)\right)$. Define

$$
K_{\lambda}^{l, r}(w, z)=\iint e^{i \lambda \tau\left[S\left(z, y_{1}\right)-S\left(w, y_{1}\right)\right]} \rho_{l}^{r}\left(w, z, \tau, y_{1}\right) d \tau d y_{1}
$$

with $\rho_{l}^{r}\left(w, z, \tau, y_{1}\right)=\rho\left(w, y_{1}, \tau\right) \gamma_{l}^{r}\left(w, y_{1}\right) \overline{\rho\left(z, y_{1}, \tau\right) \gamma_{l}^{r}\left(z, y_{1}\right)}$. The following is a reformulation of [19, Lemma 3.6].

Lemma 7.1. Fix $\lambda>1$ and $l, k$ such that $0<l<k$ and $2^{k-1}<\lambda \leq 2^{k}$, and let

$$
I_{m}^{l k}\left(z_{1}\right)=\left\{w_{1} ;\left|w_{1}-z_{1}\right| \in\left[2^{l-k} m, 2^{l-k}(m+1)\right]\right\} .
$$

Let $m \geq 2^{l \varepsilon_{1}}$ for suitably small $\varepsilon_{1}>0$ and assume that

$$
\left|S_{y_{1}}\left(w_{1}, u\left(w_{1}, z, y_{1}\right), y_{1}\right)-S_{y_{1}}\left(z_{1}, z_{2}, y_{1}\right)\right| \geq c 2^{-l}\left|w_{1}-z_{1}\right|
$$


for $w_{1} \in I_{m}^{l k}\left(z_{1}\right)$ and for $z_{1} \in I_{m}^{l k}\left(w_{1}\right)$. Then

$$
\iint_{w_{1} \in I_{m}^{l k}\left(z_{1}\right)}\left|K_{\lambda}^{l, r}(w, z)\right| d w+\iint_{z_{1} \in I_{m}^{l k}\left(w_{1}\right)}\left|K_{\lambda}^{l, r}(w, z)\right| d z \leq C_{\epsilon_{1}}(1+m)^{-1} 2^{l\left(1+\epsilon_{1}\right)} \lambda^{-2} .
$$

We shall need

Lemma 7.2. Let $N \geq 2$ and let a be a $C^{N+1}$ function on the interval $[-R, R]$, $R \leq 1$. For $l \geq 1$ let $E_{l}=\left\{t \in[-R, R]:|a(t)|>2^{-l}\right\}$ and ${ }^{c} E_{l}=[-R, R] \backslash E_{l}$. Suppose that for some $B \geq 1$

$$
\sup _{t \in[-R, R]}\left|a^{(N)}(t)\right| \leq B .
$$

Then

$$
\int_{E_{l}} \frac{\left|a^{\prime}(t)\right|}{|a(t)|} d t \leq C_{B, N} l 2^{l / N}
$$

and

$$
\int_{c_{E_{l}}}\left|a^{\prime}(t)\right| d t \leq C_{B, N} l 2^{-l(1-1 / N)} .
$$

The first inequality is proved in [19, Lemma 3.8], the second can be deduced from the first, noticing that

$$
\begin{aligned}
\int_{{ }^{c} E_{l}}\left|a^{\prime}(t)\right| d t & \leq C \sum_{m \geq 0} 2^{-l-m} \int_{E_{l+m}} \frac{\left|a^{\prime}(t)\right|}{|a(t)|} d t \\
& \leq C^{\prime} \sum_{m \geq 0} 2^{-l-m}(l+m) 2^{(l+m) / N} \leq C^{\prime \prime} l 2^{-l(1-1 / N)} .
\end{aligned}
$$

Actually by a similar argument one could also obtain the first inequality from the second.

Proof of Lemma 7.1. By symmetry it suffices to consider the first term in (7.3), for fixed $z_{1}$. In (7.1) we perform first one integration by parts with respect to $y_{1}$ and then many integrations by parts with respect to $\tau$. Let $\mathcal{L}$ be the differential operator defined by

$$
\mathcal{L} g\left(y_{1}, \tau\right)=\frac{1-i \lambda \tau\left(S_{y_{1}}^{\prime}\left(z, y_{1}\right)-S_{y_{1}}^{\prime}\left(w, y_{1}\right)\right) g_{y_{1}}^{\prime}}{1+\left|\lambda^{2} \tau^{2}\left[S_{y_{1}}^{\prime}\left(z, y_{1}\right)-S_{y_{1}}^{\prime}\left(w, y_{1}\right)\right]\right|^{2}}
$$

and ${ }^{t} \mathcal{L}$ its formal transpose. Then integration by parts yields

$$
K_{\lambda}^{l, r}(w, z)=\int e^{i \lambda \tau\left[S\left(z, y_{1}\right)-S\left(w, y_{1}\right)\right]} \frac{\left(1-\frac{\partial^{2}}{(\partial \tau)^{2}}\right)^{N}\left[{ }^{t} \mathcal{L} \gamma_{l}^{r}\right]\left(w, z, \tau, y_{1}\right)}{\left(1+\lambda^{2}\left|S\left(w, y_{1}\right)-S\left(z, y_{1}\right)\right|^{2}\right)^{N}} d \tau d y_{1} .
$$

Let

$$
J_{l r}(w, z)=\left\{y_{1} ; \gamma_{l}^{r}\left(w, y_{1}\right) \gamma_{l}^{r}\left(z, y_{1}\right) \neq 0\right\}
$$

and for $0 \leq j \leq n$

$$
\begin{aligned}
& J_{l r}^{j 1}(w, z)=\left\{y_{1} \in J_{l r}(w, z) ; \frac{\partial}{\partial y_{1}}\left[\chi_{l}^{r_{j}}\left(\sigma_{j}\left(w, y_{1}\right)\right)\right] \neq 0\right\}, \\
& J_{l r}^{j 2}(w, z)=\left\{y_{1} \in J_{l r}(w, z) ; \frac{\partial}{\partial y_{1}}\left[\chi_{l}^{r_{j}}\left(\sigma_{j}\left(z, y_{1}\right)\right)\right] \neq 0\right\} .
\end{aligned}
$$


Arguing as in [19] one obtains the estimate

$$
\begin{aligned}
\left|K_{\lambda}^{l, r}(w, z)\right| & \leq C\left[\int_{J_{l r}(w, z)} H_{\lambda, 1}^{l, r}\left(w, z, y_{1}\right) d y_{1}+\int_{J_{l r}(w, z)} H_{\lambda, 2}^{l, r}\left(w, z, y_{1}\right) d y_{1}\right. \\
& \left.+\sum_{j=0}^{n} \int_{J_{l r}^{j 1}(w, z)} H_{\lambda, 3}^{l, r, j}\left(w, z, y_{1}\right) d y_{1}+\sum_{j=0}^{n} \int_{J_{l r}^{j 2}(w, z)} H_{\lambda, 4}^{l, r, j}\left(w, z, y_{1}\right) d y_{1}\right]
\end{aligned}
$$

where

$$
\begin{aligned}
H_{\lambda, 1}^{l, r}\left(w, z, y_{1}\right) & =\frac{1}{\left(1+\lambda\left|S_{y_{1}}^{\prime}\left(w, y_{1}\right)-S_{y_{1}}^{\prime}\left(z, y_{1}\right)\right|\right)\left(1+\lambda^{2}\left|S\left(w, y_{1}\right)-S\left(z, y_{1}\right)\right|^{2}\right)^{N}}, \\
H_{\lambda, 2}^{l, r}\left(w, z, y_{1}\right) & =\frac{\lambda\left|S_{y_{1} y_{1}}^{\prime \prime}\left(w, y_{1}\right)-S_{y_{1} y_{1}}^{\prime \prime}\left(z, y_{1}\right)\right|}{1+\lambda^{2}\left|S_{y_{1}}^{\prime}\left(w, y_{1}\right)-S_{y_{1}}^{\prime}\left(z, y_{1}\right)\right|^{2}} \frac{1}{\left(1+\lambda^{2}\left|S\left(w, y_{1}\right)-S\left(z, y_{1}\right)\right|^{2}\right)^{N}}, \\
H_{\lambda, j, 3}^{l, r}\left(w, z, y_{1}\right) & =\frac{2^{l-r_{j}}\left|\partial_{y_{1}} \sigma_{j}\left(w, y_{1}\right)\right|}{\left(1+\lambda\left|S_{y_{1}}^{\prime}\left(w, y_{1}\right)-S_{y_{1}}^{\prime}\left(z, y_{1}\right)\right|\right)\left(1+\lambda^{2}\left|S\left(w, y_{1}\right)-S\left(z, y_{1}\right)\right|^{2}\right)^{N}}, \\
H_{\lambda, j, 4}^{l, r}\left(w, z, y_{1}\right) & =\frac{2^{l-r_{j}}\left|\partial_{y_{1}} \sigma_{j}\left(z, y_{1}\right)\right|}{\left(1+\lambda\left|S_{y_{1}}^{\prime}\left(w, y_{1}\right)-S_{y_{1}}^{\prime}\left(z, y_{1}\right)\right|\right)\left(1+\lambda^{2}\left|S\left(w, y_{1}\right)-S\left(z, y_{1}\right)\right|^{2}\right)^{N}} .
\end{aligned}
$$

We now estimate separately each term in (7.4). For fixed $z$ set $u\left(w_{1}, y_{1}\right):=$ $v\left(w_{1}, z, y_{1}\right)$. In the argument to follow the constants in the various estimates will be chosen independently of $z$. Integrations will always be performed over subsets of a fixed compact set, in particular we shall always assume that $w_{1} \in I_{m}^{l k}\left(z_{1}\right)$, so that $\lambda\left|w_{1}-z_{1}\right| \approx 2^{l} m$, moreover the length of $I_{m}^{l k}\left(z_{1}\right)$ is $\approx 2^{l} \lambda^{-1}$. Then

$$
\left|S_{y_{1}}^{\prime}\left(w, y_{1}\right)-S_{y_{1}}^{\prime}\left(z, y_{1}\right)\right| \geq c_{0}\left[2^{-l}\left|w_{1}-z_{1}\right|-C_{0}\left|w_{2}-u\left(w_{1}, y_{1}\right)\right|\right]
$$

hence

$$
\left|S_{y_{1}}^{\prime}\left(w, y_{1}\right)-S_{y_{1}}^{\prime}\left(z, y_{1}\right)\right| \geq c^{\prime} \lambda^{-1}(m+1) \quad \text { if }\left|w_{2}-u\left(w_{1}, y_{1}\right)\right| \leq C_{0}^{-1} 2^{-l}\left|w_{1}-z_{1}\right| \text {. }
$$

Note also that

$$
\begin{aligned}
& \int_{\substack{\left|w_{2}-u\left(w_{1}, y_{1}\right)\right| \\
\geq C_{0}^{-1} 2^{-l}\left|w_{1}-z_{1}\right|}}\left(1+\lambda\left|w_{2}-u\left(w_{1}, y_{1}\right)\right|\right)^{-2 N} d w_{2} d y_{1} d w_{1} \\
& \leq C \lambda^{-2 N}\left(\left|w_{1}-z_{1}\right| 2^{-l}\right)^{1-2 N} \leq C^{\prime} m^{1-2 N} \lambda^{-1}
\end{aligned}
$$

It follows that

$$
\begin{aligned}
& \iiint_{\substack{w_{1} \in I_{m}^{l k}\left(z_{1}\right) \\
y_{1} \in J_{l r}(w, z)}} H_{\lambda, 1}^{l, r}\left(w, z, y_{1}\right) d y_{1} d w \\
& \leq C\left[\int \frac{2^{l}}{\lambda\left|w_{1}-z_{1}\right|} \iint\left(1+\lambda\left|w_{2}-u\left(w_{1}, y_{1}\right)\right|\right)^{-2 N} d w_{2} d y_{1} d w_{1}\right. \\
& \left.\quad+\iiint \int_{\left|w_{2}-u\left(w_{1}, y_{1}\right)\right|}\left(1+\lambda\left|w_{2}-u\left(w_{1}, y_{1}\right)\right|\right)^{-2 N} d w_{2} d y_{1} d w_{1}\right] \\
& \quad \underset{C_{0}^{-1} 2^{-l}\left|w_{1}-z_{1}\right|}{\leq C\left[(1+m)^{-1}+m^{1-2 N}\right] 2^{l} \lambda^{-2} .}
\end{aligned}
$$


In order to estimate the second term in (7.4) we wish to apply Lemma 7.2 (with $N \gg 1 / \epsilon_{2}$, some $\left.\epsilon_{2} \in\left(0, \varepsilon_{1}\right)\right)$ to the function

$$
y_{1} \mapsto a\left(w_{1}, y_{1}\right)=\frac{S_{y_{1}}^{\prime}\left(w_{1}, u\left(w_{1}, y_{1}\right), y_{1}\right)-S_{y_{1}}^{\prime}\left(z, y_{1}\right)}{w_{1}-z_{1}} .
$$

We have $\left|a\left(w_{1}, y_{1}\right)\right| \geq c 2^{-l}$ for $w_{1} \in I_{m}^{l k}\left(z_{1}\right)$ and

$$
\left|a\left(w_{1}, y_{1}\right)\left(w_{1}-z_{1}\right)-\left[S_{y_{1}}^{\prime}\left(w, y_{1}\right)-S_{y_{1}}^{\prime}\left(z, y_{1}\right)\right]\right| \leq C_{1}\left|w_{2}-u\left(w_{1}, y_{1}\right)\right| .
$$

Moreover

$$
\begin{aligned}
& S_{y_{1} y_{1}}^{\prime \prime}\left(w, y_{1}\right)-S_{y_{1} y_{1}}^{\prime \prime}\left(z, y_{1}\right) \\
& =\partial_{y_{1}} a\left(w_{1}, y_{1}\right)\left(w_{1}-z_{1}\right)-S_{y_{1} x_{2}}^{\prime \prime}\left(w_{1}, u\left(w_{1}, y_{1}\right), y_{1}\right) \partial_{y_{1}} u\left(w_{1}, y_{1}\right) \\
& \quad+S_{y_{1} y_{1}}^{\prime \prime}\left(w, y_{1}\right)-S_{y_{1} y_{1}}^{\prime \prime}\left(w_{1}, u\left(w_{1}, y_{1}\right), y_{1}\right) \\
& =\partial_{y_{1}} a\left(w_{1}, y_{1}\right)\left(w_{1}-z_{1}\right)+O\left(\left|S_{y_{1}}^{\prime}\left(w, y_{1}\right)-S_{y_{1}}^{\prime}\left(w_{1}, u\left(w_{1}, y_{1}\right), y_{1}\right)\right|\right) \\
& \quad+O\left(w_{2}-u\left(w_{1}, y_{1}\right)\right) .
\end{aligned}
$$

Here we have used that $\partial_{y_{1}} u=O\left(\left|S_{y_{1}}^{\prime}\left(w, y_{1}\right)-S_{y_{1}}^{\prime}\left(w_{1}, u\left(w_{1}, y_{1}\right), y_{1}\right)\right|\right)$; this follows by differentiation of the identity $S\left(w_{1}, u\left(w_{1}, y_{1}\right), y_{1}\right)-S\left(z, y_{1}\right)=0$. Consequently if $\left|w_{2}-u\right| \leq c 2^{-l}\left|w_{1}-z_{1}\right|$ for small $c$, then

$$
\begin{aligned}
& \frac{\lambda\left|S_{y_{1} y_{1}}^{\prime \prime}\left(w, y_{1}\right)-S_{y_{1} y_{1}}^{\prime \prime}\left(z, y_{1}\right)\right|}{1+\lambda^{2}\left|S_{y_{1}}^{\prime}\left(w, y_{1}\right)-S_{y_{1}}^{\prime}\left(z, y_{1}\right)\right|^{2}} \leq \\
& C \frac{2^{l}}{\lambda\left|w_{1}-z_{1}\right|}\left[\frac{\left|\partial_{y_{1}} a\left(w_{1}, y_{1}\right)\right|}{\left|a\left(w_{1}, y_{1}\right)\right|}+1+\lambda\left|w_{2}-u\left(w_{1}, y_{1}\right)\right|\right],
\end{aligned}
$$

while in the case $\left|w_{2}-u\right| \geq c 2^{-l}\left|w_{1}-z_{1}\right|$ we have

$$
\frac{\lambda\left|S_{y_{1} y_{1}}^{\prime \prime}\left(w, y_{1}\right)-S_{y_{1} y_{1}}^{\prime \prime}\left(z, y_{1}\right)\right|}{1+\lambda^{2}\left|S_{y_{1}}^{\prime}\left(w, y_{1}\right)-S_{y_{1}}^{\prime}\left(z, y_{1}\right)\right|^{2}} \leq C \lambda\left[\left|w_{1}-z_{1}\right|+C^{\prime}\left|w_{2}-u\left(w_{1}, y_{1}\right)\right|\right] .
$$

Therefore using Lemma 7.2 we obtain

$$
\begin{aligned}
& \iiint_{\substack{w_{1} \in I_{m}^{l k}\left(z_{1}\right) \\
y_{1} \in J_{l r}(w, z)}} H_{\lambda, 2}^{l, r}\left(w, z, y_{1}\right) d y_{1} d w \\
& \leq C \int \frac{2^{l}}{\lambda\left|w_{1}-z_{1}\right|} \int\left[\frac{\left|\partial_{y_{1}} a\left(w_{1}, y_{1}\right)\right|}{\left|a\left(w_{1}, y_{1}\right)\right|}+1\right] \int \frac{d w_{2} d y_{1} d w_{1}}{\left(1+\lambda\left|w_{2}-u\left(w_{1}, y_{1}\right)\right|\right)^{2 N-1}} \\
& \quad+C \int_{\substack{\left|w_{1}-z_{1}\right| \\
\approx 2^{l} \lambda^{-1} m}} \int_{\substack{\left|w_{2}-u\right| \geq \\
c 2^{-l}\left|w_{1}-z_{1}\right|}} \frac{2^{l} m}{\left(1+\lambda\left|w_{2}-u\left(w_{1}, y_{1}\right)\right|\right)^{2 N-1}} d w_{2} d y_{1} d w_{1} \\
& \leq C\left(\epsilon_{2}\right) 2^{l\left(1+\epsilon_{2}\right)} \lambda^{-2} m^{-1}+C^{\prime} 2^{2 l} \lambda^{-2} m^{3-2 N}
\end{aligned}
$$

where $\epsilon_{2}>0$ is arbitrary (we choose $\epsilon_{2}<\epsilon_{1}$ ). Since we assume that $m \geq 2^{l \epsilon_{1}}$ we may choose $N$ large enough to obtain the desired estimate for $H_{\lambda, 2}^{l, r}\left(w, z, y_{1}\right)$.

The estimate for the term $H_{\lambda, 3}^{l, r, j}\left(w, z, y_{1}\right)$ is similar. This time we shall apply Lemma 7.2 to the function

$$
y_{1} \mapsto \alpha_{j}\left(w_{1}, y_{1}\right)=\sigma_{j}\left(w_{1}, u\left(w_{1}, y_{1}\right), y_{1}\right) .
$$


Note that

$$
\begin{aligned}
\partial_{y_{1}} \sigma_{j}\left(w, y_{1}\right) & =\partial_{y_{1}} \alpha_{j}\left(w, y_{1}\right)-\partial_{w_{2}} \sigma_{j}\left(w_{1}, u\left(w_{1}, y_{1}\right), y_{1}\right) \partial_{y_{1}} u \\
& +\partial_{y_{1}} \sigma\left(w, y_{1}\right)-\partial_{y_{1}} \sigma\left(w_{1}, u\left(w_{1}, y_{1}\right), y_{1}\right)
\end{aligned}
$$

and therefore

$$
\begin{aligned}
& \left|\partial_{y_{1}} \sigma_{j}\left(w, y_{1}\right)-\partial_{y_{1}} \alpha_{j}\left(w_{1}, y_{1}\right)\right| \\
& \quad \leq C_{0}\left[\left|S_{y_{1}}^{\prime}\left(w, y_{1}\right)-S_{y_{1}}^{\prime}\left(w_{1}, u\left(w_{1}, y_{1}\right), y_{1}\right)\right|+\left|w_{2}-u\left(w_{1}, y_{1}\right)\right|\right]
\end{aligned}
$$

and

$$
\left|\sigma_{j}\left(w, y_{1}\right)-\alpha_{j}\left(w_{1}, y_{1}\right)\right| \leq C_{0}\left|w_{2}-u\left(w_{1}, y_{1}\right)\right| .
$$

In the support of $\partial_{y_{1}} \chi_{l}^{r}\left(\sigma_{j}\left(w, y_{1}\right)\right)$ the function $\sigma_{j}\left(w, y_{1}\right)$ is bounded above by $C^{\prime} 2^{l-r_{i}}$. Therefore we have either $\left|\alpha_{j}\left(w_{1}, y_{1}\right)\right| \leq C^{\prime} 2^{l-r_{i}+1}$ or $\left|w_{2}-u\left(w_{1}, y_{1}\right)\right| \geq$ $c\left|\alpha_{j}\left(w_{1}, y_{1}\right)\right| \geq C^{\prime} 2^{l-r_{i}+1}$. Consequently

$$
\begin{aligned}
& \iiint_{\substack{w_{1} \in I_{m}^{l k}\left(z_{1}\right) \\
y_{1} \in J_{l r}(w, z)}} H_{\lambda, 3}^{l, r, j}(w, z) d y_{1} d w \\
& \leq C\left[\int \frac{2^{l}}{\lambda\left|w_{1}-z_{1}\right|} \int_{\left|\alpha_{j}\right| \leq C 2^{-l+r_{i}}} \int \frac{1+2^{l-r_{i}}\left|\partial_{y_{1}} \alpha_{j}\left(w_{1}, y_{1}\right)\right|}{\left(1+\lambda\left|w_{2}-u\left(w_{1}, y_{1}\right)\right|\right)^{2 N-1}} d w_{2} d y_{1} d w_{1}\right. \\
& \quad+\int \frac{2^{l}}{\lambda\left|w_{1}-z_{1}\right|} \int_{\left|\alpha_{j}\right| \geq C 2^{-l+r_{i}}} \int_{\left|w_{2}-u\right| \geq} \frac{1+2^{l-r_{i}}\left|\partial_{y_{1}} \alpha_{j}\left(w_{1}, y_{1}\right)\right|}{\left(1+\lambda\left|w_{2}-u\left(w_{1}, y_{1}\right)\right|\right)^{2 N-1}} d w_{2} d y_{1} d w_{1} \\
& \quad+C \int_{\substack{\left|w_{1}-z_{1}\right| \\
\approx^{l} \lambda^{-1} m}} \int_{\substack{\left|w_{2}-u\right| \geq \\
c 2^{-l}\left|w_{1}-z_{1}\right|}} \frac{2^{l} m}{\left(1+\lambda\left|w_{2}-u\left(w_{1}, y_{1}\right)\right|\right)^{2 N-1}} d w_{2} d y_{1} d w_{1} .
\end{aligned}
$$

We first perform the $w_{2}$ integration, then use the second Lemma and we obtain, by a straightforward computation, the bound (7.9) for the sum of the three previous expressions. The analogous estimate for $H_{\lambda, 4}^{l, r, j}\left(w, z, y_{1}\right)$ is similar (actually simpler, since the terms $\sigma_{j}\left(z_{1}, y_{1}\right)$ do not vary in $\left.w\right)$. The proof is concluded by combining the desired estimates for the terms in (7.4).

\section{REFERENCES}

1. M. Christ, Hilbert transforms along curves, I. Nilpotent groups, Ann. Math. 122 (1985), 575-596. MR 87f:42039a

2. differential equations, ed. by J. Garcia-Cuerva, E. Hernandez, F. Soria and J. L. Torrea, CRC Press, 1995. MR 97e:44007

3. M. Christ, A. Nagel, E. M. Stein and S. Wainger, Singular and maximal Radon transforms: analysis and geometry, preprint, 1997 (to appear).

4. A. Greenleaf and A. Seeger, Fourier integral operators with fold singularities, J. reine ang. Math. 455 (1994), 35-56. MR 95h:58130

5. _ Fourier integral operators with simple cusps, Amer. J. Math. (to appear).

6. A. Greenleaf and G. Uhlmann, Composition of some singular Fourier integral operators and estimates for the X-ray transform, I, Ann. Inst. Fourier (Grenoble) 40 (1990), 443-466. MR 91k:58126

7. Composition of some singular Fourier integral operators and estimates for the X-ray transform, II, Duke Math. J. 64 (1991), 413-419. MR 93b:58146 
8. V. Guillemin, Cosmology in $(2+1)$-dimensions, cyclic models and deformations of $M_{2,1}$, Ann. of Math. Studies 121, Princeton Univ. Press, Princeton, 1989. MR 91k:58140

9. L. Hörmander, Hypoelliptic second order differential equations, Acta Math. 119 (1967), 147171. MR 36:5526

10. __ Fourier integral operators I, Acta Math. 127 (1971), 79-183. MR 52:9299

11. J. J. Kohn, Boundary behavior of $\bar{\partial}$ on weakly pseudo-convex manifolds of dimension two, J. Diff. Geom. 6 (1972), 523-542. MR 48:727

12. R. Melrose and M. Taylor, Near peak scattering and the correct Kirchhoff approximation for a convex obstacle, Adv. in Math. 55 (1985), 242-315. MR 86m:35095

13. D. H. Phong, Singular integrals and Fourier integral operators, Essays on Fourier analysis in honor of Elias M. Stein, edited by C. Fefferman, R. Fefferman and S. Wainger, Princeton University Press, 1995. MR 95k:42026

14. D. H. Phong and E. M. Stein, Hilbert integrals, singular integrals and Radon transforms I, Acta Math. 157 (1986), 99-157. MR 88i:42028a

15. , Radon transforms and torsion, International Mathematics Research Notices (1991), 49-60. MR 93g:58144

16. 140 (1994), 703-722. MR 96c:35206

17. 146-177. CMP 98:05

18. L. P. Rothschild and E. M. Stein, Hypoelliptic operators and nilpotent groups, Acta Math. 137 (1976), 247-320. MR 55:9171

19. A. Seeger, Degenerate Fourier integral operators in the plane, Duke Math. J. 71 (1993), 685-745. MR 94h:35292

20. H. Smith and C. D. Sogge, $L^{p}$ regularity for the wave equation with strictly convex obstacles, Duke Math. J. 73 (1994), 97-153. MR 95c:35048

21. C. D. Sogge and E. M. Stein, Averages of functions over hypersurfaces: smoothness of generalized Radon transforms, J. Analyse Math. 54 (1990), 165-188. MR 91i:58145

22. E. M. Stein, Harmonic analysis: Real variable methods, orthogonality and oscillatory integrals, Princeton Univ. Press, 1993. MR 95c: 42002

23. S. Sternberg, Differential Geometry, second edition, Chelsea, 1983. MR 88f:58001

Department of Mathematics, University of Wisconsin, Madison, Wisconsin 53706

E-mail address: seeger@math.wisc.edu 